\title{
3D wake dynamics of the VAWT: experimental and numerical investigation
}

\author{
Carlos Ferreira ${ }^{*}$ Claudia Hofemann $†$ Kristian Dixon $\ddagger$ \\ Gijs van Kuik §, Gerard van Bussel \\ Delft University of Technology, Delft, 2629 HS, The Netherlands
}

The Vertical Axis Wind Turbine, in its $2 D$ form, is characterized by a complex unsteady aerodynamic flow, including dynamic stall and blade vortex interaction.

Adding to this complexity, the $3 D$ flow causes spanwise effects and the presence of trailing vorticity and tip vortices.

The objective of the current paper is to bring insight into the $3 D$ development of the near wake of a H-VAWT, understanding:

- The spanwise blade load distribution in the upwind and the downwind blade passages.

- The trajectory of tip vortices, including the inboard movement and the radial expansion of the shed and the trailing vorticity.

- The impact of the $3 D$ flow phenomena on the efficiency of the VAWT.

- The blade vortex interaction of the upwind tip vortex with the downwind blade passage.

- The induction due to trailing vorticity.

The investigation is composed of experimental wind tunnel research with Stereo-PIV and modeling of the rotor and wake with a $3 D$ unsteady panel method.

A two bladed H-Darrieus VAWT model is tested in the low speed/low turbulence wind tunnel at Delft University of Technology. Stereo-PIV measurements are used to visualize the flow in the near wake focusing on the flow field around four tip geometries. The measurement planes cover several sections of the rotor volume, allowing for the reconstruction of the evolution of the tip vortex. The formation, the convection and the dissipation for each tip vortex are quantified.

The experimental PIV data is used to validate the 3D, unsteady, multi-body, free-wake panel method.

The combination of the results of the panel model validated by experiments, in particular the Stereo PIV results, allows to understand the impact of the near wake development on the upwind blade passage, as well as the energy conversion process during the downwind blade passage.

\section{Nomenclature}

induction factor in the $x$-direction (aligned with $U_{\infty}$ )

aerofoil/blade chord, $m$

$D \quad$ rotor diameter, $m$

$F_{N}$

rotor height/blade span, $m$

rotor radius, $m$

${ }^{*}$ DUWIND, TUDelft, The Netherlands.

${ }^{\dagger}$ DUWIND, TUDelft, The Netherlands.

${ }^{\ddagger}$ DUWIND, TUDelft, The Netherlands

§Professor, DUWIND, TUDelft, The Netherlands.

IProfessor, DUWIND, TUDelft, Kluyverweg 1, 2629 HS, Delft, The Netherlands 


$\begin{array}{ll}U_{\infty} & \text { unperturbed velocity, } \mathrm{m} / \mathrm{s} \\ U_{x} & \text { local velocity in the } x \text {-direction, } \mathrm{m} / \mathrm{s} \\ U_{\text {ind }(x)} & \text { induced velocity in the } x \text {-direction, } \mathrm{m} / \mathrm{s} \\ x, y, z & \text { space coordinates, } \mathrm{m} \\ z_{\text {tip }} & z \text { coordinate measured from blade tip, } \mathrm{m} \\ \Gamma & \text { circulation, } \mathrm{m}^{2} / \mathrm{s} \\ \theta & \text { azimuth angle, } \\ \lambda & \text { tip-speed ratio, } \omega R / U_{\infty} \\ \rho & \text { air density, } \mathrm{kg} / \mathrm{m}^{3} \\ \omega & \text { angular velocity, } \mathrm{rad} / \mathrm{s} \\ \Omega & \text { vorticity, } \mathrm{s}^{-1}\end{array}$

\section{Introduction}

A VAWT is a tri-dimensional rotor, with a finite blade span/rotor height. This finite span dimension implies a spanwise circulation distribution, and thus trailing vorticity.

The $3 D$ geometry of the lift-driven Vertical Axis Wind Turbine rotor design is highly determined by the spanwise blade curvature. The blade shape curvature design is constrained by the rotor area and centrifugal and gravity loads; for the large VAWT, the design shape has evolved to catenary, parabolic and troposkien blade shapes (see, ${ }^{1},{ }^{2},{ }^{3},{ }^{4},{ }^{56}$ and $^{7}$ ).

For small wind turbines, current designs tend to opt for the straight-bladed H-VAWT $\left(\mathrm{see}^{8}\right)$. These HVAWT have several design variables, including rotor twist, transition of blade to support strut, and blade-tip shape.

In this work we use the straight H-VAWT, since it is an interesting study case for aerodynamics analysis:

- The curvature of the blade and rotor twist is null (thus eliminating two variables), with the geometry being defined by the aspect ratio of the blade $(H / c)$ and the aspect ratio of the rotor $(H / D)$. The rotor is defined by a minimum number of parameters, making it more suitable for analysis.

- The spanwise circulation distribution, at each azimuthal position, is a function solely of the height of the rotor, aspect ratio of the blade and local blade section (no blade curvature and/or twist effects)

- The cylindrical rotor volume allows for the analysis of the convection of the tip vortex and its interaction with the downwind passage.

In the Horizontal Axis Wind Turbine, the wake is (mainly) composed of trailing vorticity (under uniform, steady flow conditions), of which the tip vortex is usually the strongest element; the trailing vorticity of the HAWT is a consequence of the exchange of energy at the rotor.

In the VAWT, the exchange of energy is a $2 D$ phenomenon, expressed by the distribution of shed vorticity, and the existence of trailing vorticity (and thus, tip vortices) is not required. Consequently, in the VAWT, the trailing vorticity and the tip vortex, in particular, are inefficiencies of the energy conversion process.

This inefficiency is expressed by the added induction due to the trailing vorticity, which increases the induction factor at the rotor, and by the influence of the tip vortex on the definition of the blade-wake interaction region during the downwind passage of the blade.

Therefore, for the $3 D$ rotor, it is important to understand the generation and convection of the trailing vorticity, its impact on the induction field, blade-wake interaction and the energy conversion at the rotor scale.

The tip vortex is the most interesting element of trailing vorticity to analyze, since:

- It is, for conventional blade/rotor design, the strongest element of trailing vorticity, with a scale comparable to the maximum bound circulation on the blade.

- It delimits the wake the in spanwise direction, thus the convection of the tip vortex expresses the spanwise convection of the wake.

- The convection of the tip vortex, contrary to what happens in the HAWT, does not necessarily have to occur in an outboard direction $\left(\frac{2\|z\|}{H}>1\right)$. 
- Due to its strength, the interaction between the tip vortex and the blade is particularly relevant.

\section{Experimental measurements with (Stereo) Particle Image Velocimetry}

The experimental campaign measures the evolution of the upwind-generated wake using (Stereo) Particle Image Velocimetry. The data obtained with Stereo-PIV allows a detailed measurement of the convection of the tip vortex, the variation of tip vortex strength with azimuth angle and the midspan wake convection, for normal and skewed flow.

\section{A. Rotor model and experimental setup}

The model is a two-bladed H-VAWT, $0.285 \mathrm{~m}$ in radius, $0.7 \mathrm{~m}$ in height, and $0.06 \mathrm{~m}$ in chord at midspan. Each blade is constructed with a different profile, either a NACA0015 or a NACA0018 cross section. The use of the different profiles is to enable the analysis of the impact of blade thickness at the midspan section, a result not discussed in this work.

The different tip shapes were developed to evaluate the effect of tip shape on the strength and location of the tip vortex, a result also not discussed in this work.

The NACA0015 blade has two different blade tips. The first is a straight blade tip, which we will refer to as a straight tip or flat tip $(F L)$. The second has an elliptic chord distribution, starting at a distance from the tip of $1 c=0.086 H$. The elliptic distribution ends at the tip with a local chord $\frac{1}{3} c$, where $c$ is the blade chord at midspan. The NACA0015 profile shape is maintained throughout the tip. The elliptic chord distribution is defined by keeping the trailing edge as a straight line; we will refer to this tip as the trailing edge tip (TE). A planform view of the NACA0015 blade is shown in Figure 1a.

The NACA0018 profile blade also has two different blade tips, both with an elliptic chord distribution as described previously for the NACA0015 blade. However, one of the tips is defined by keeping the quarterchord line straight (we refer to this tip as the quarter-chord tip, $Q C$ ), while the other is defined by keeping the leading edge line straight (we refer to this tip as the leading edge tip, LE). A planform view of the $N A C A 0018$ blade is shown in Figure 1b, , with the $L E$ tip on the left and the $Q C$ tip on the right.

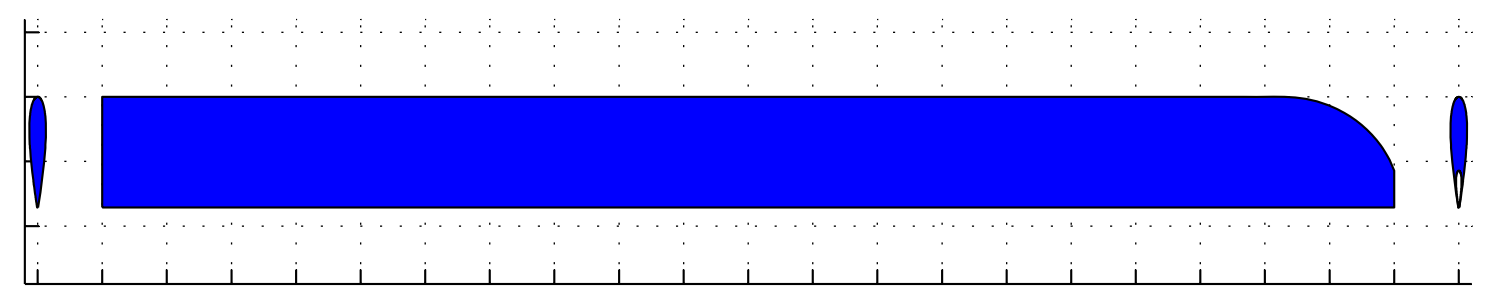

(a) NACA0015 blade.

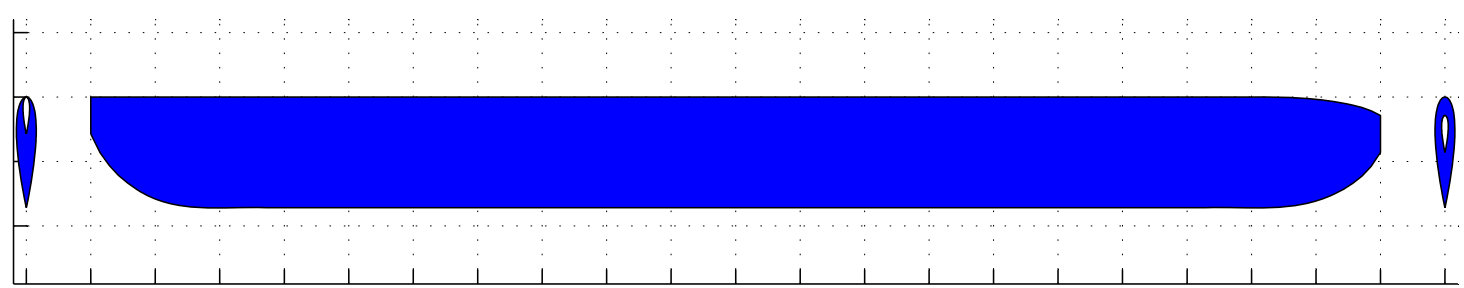

(b) NACA0018 blade.

Figure 1: Planform and side views of the two rotor blades.

The experiment was conducted in the Low Speed Low Turbulence Wind Tunnel of the TUDelft. The measurements presented are at conditions $U_{\infty}=5 \mathrm{~m} / \mathrm{s}$ and $\lambda=4$.

The reader is referred to the master thesis of ${ }^{9}$ for details regarding the experimental setup, the results for the evolution and dissipation of the tip-vortex strength, the method of calculation of the tip-vortex circulation, the uncertainty of the measured data and measurements at other tip-speed ratios. 


\section{B. Measurement planes and fields of view}

The measurements planes/fields of view were set at two different orientations, thus obtaining two different perspectives of the flow field.

Figure 2a shows a schematic representation of the rotor (blades and swept surface) and the fields of view for the data acquired and used in the analysis presented in Section C. The fields of view are aligned parallel to the $x y$-plane, spaced $1 / 6 c$ apart in the $z$-direction; the phase-locked averaged three-component velocity fields, measured at the fourteen planes, are combined in Section $\mathrm{C}$ to create the vorticity iso-surfaces of the tip vortex at the several azimuthal positions.

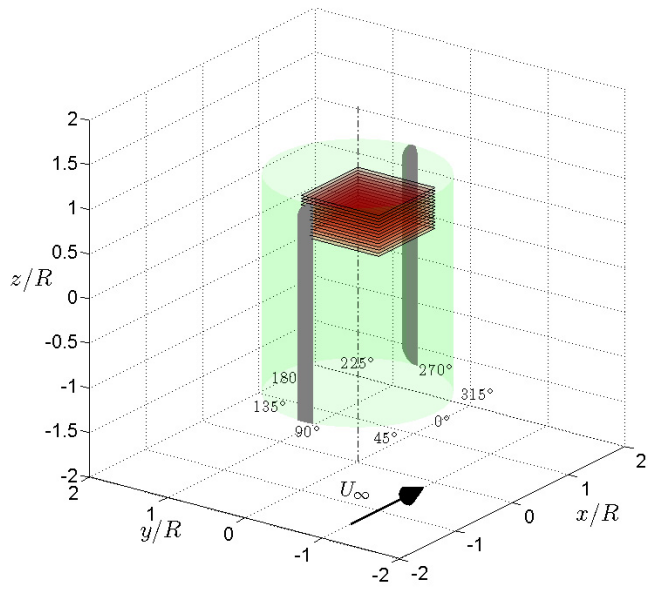

(a) Different fields of view (laser sheet location), along span. Planes spaced in the $z$-direction $\Delta z / c=$ $1 / 6$, on a range $0.68<\frac{2 z}{H}<1.06$.

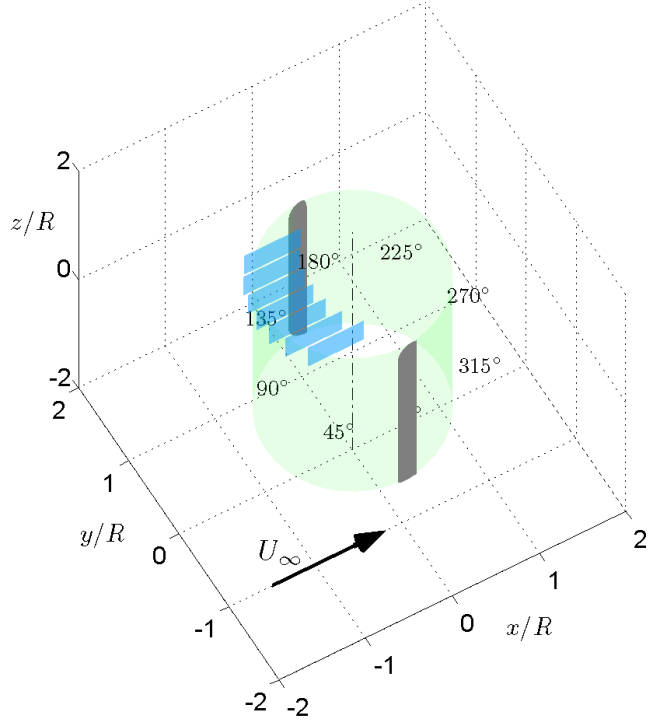

(b) Different fields of view, along the $y$-direction, $\Delta y / R=0.21$, on a range $-0.63<\frac{y}{R}<0.63$.

Figure 2: Planes of the different fields of view for the PIV measurements.

Figure $2 \mathrm{~b}$ shows a schematic representation for the second orientation of the field-of-view planes, parallel to the $x z$-plane, at $y / R=-0.63,-0.42,-0.21,0 ., 0.21,0.42$, and $y / R=0.63$. Data from these planes are used in Sections D for determining the location of the tip-vortex core and tip-vortex strength (circulation).

\section{C. $3 D$ vorticity iso-surface representation}

Figures 3 to 6 are constructed from combining and interpolating the phase-locked average velocity measurements at the different planes/fields of view described in Figure 2a.

Figure 3 shows two views of the $3 D$ vorticity iso-surface generated by the trailing edge blade tip $(T E)$, when the blade is at $\theta=110^{\circ}$, with vorticity value $\frac{\Omega c}{U_{\infty}}=5$; the figure shows wake segment generated during the most upwind moment of generation of the wake $\left(85^{\circ}<\theta<100^{\circ}\right.$, considering the location from leading edge to trailing edge), a region where the strength of the tip-vortex segment is theoretically maximum.

The iso-surface shows not only the tip vortex, but also the adjacent wake and the curvature of the adjacent wake as it is rolled up by the tip vortex. However, for the VAWT, the curvature of the wake and the induction that the tip vortex exerts over the adjacent wake has further implications. The tip vortex travels downwind faster than the remaining wake, thus (theoretically) reaching the downwind blade passage first.

A difference between the tip vortex (and the adjacent wake) of the VAWT and that of a HAWT or wing in steady flight is the importance of the shed vorticity. For the wing in steady flight, the induction (downwash) is strongest close to the tip vortex, since the induction is determined by the trailing vorticity, of which the tip vortex is the strongest element.

For the VAWT, on the other hand, the induction due to shed vorticity is similar to that due to trailing vorticity, resulting in an increased induction near the midspan of the blade. 


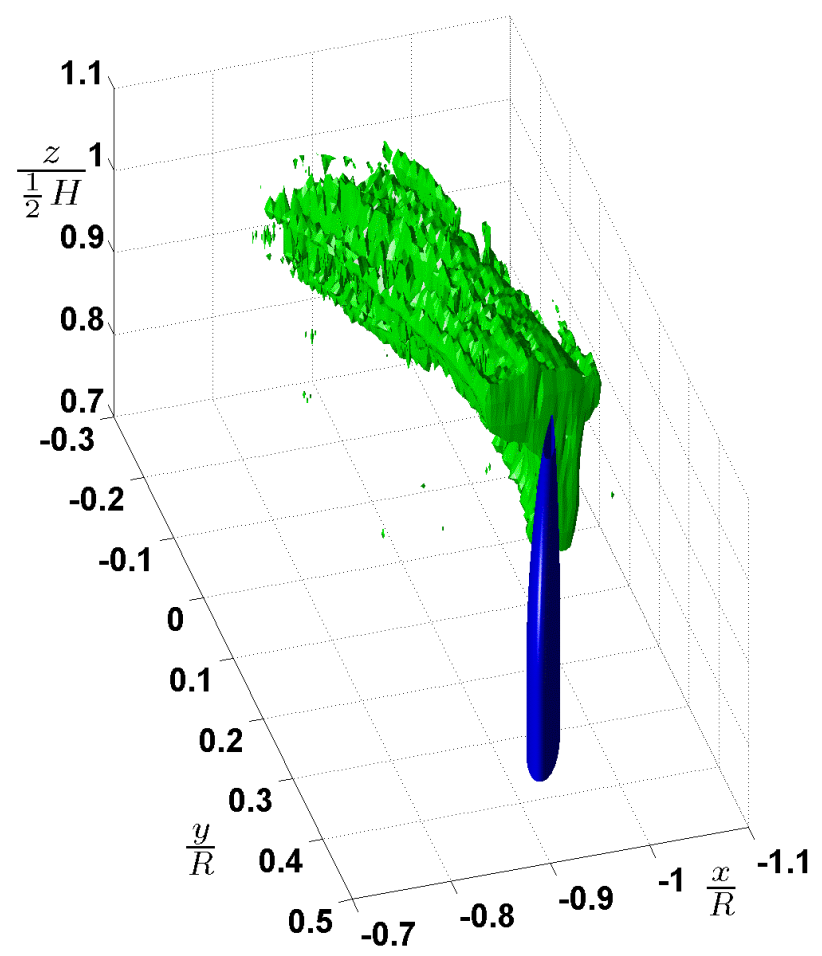

(a) View a).

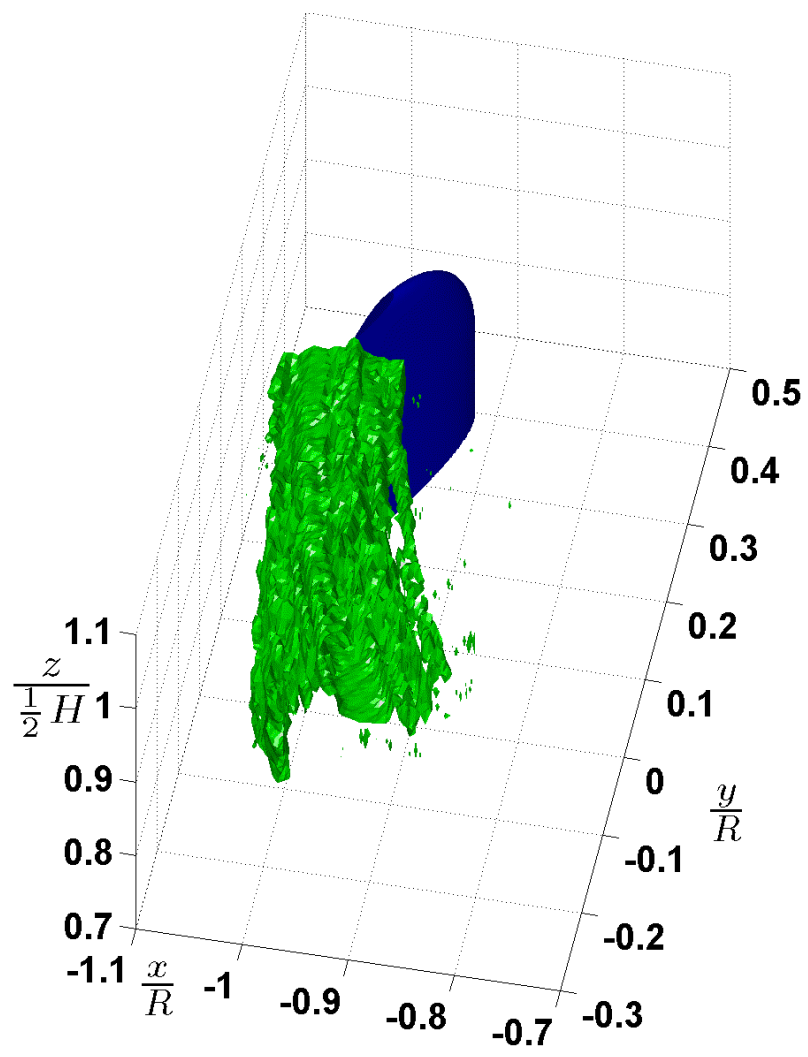

(b) View b).

Figure 3: $3 D$ reconstruction of the vorticity iso-surface $\left(\frac{\Omega c}{U_{\infty}}=5\right)$, at $\theta=110^{\circ}$.

5 of 33 
Figure 4 shows the same case as Figure 3 for higher vorticity value $\left(\frac{\Omega c}{U_{\infty}}=30\right)$, describing an iso-surface closer to the vortex core. The figure gives a clear detail of the inboard $\left(\left\|\frac{2 z}{H}\right\|<1\right)$ movement of the tip vortex as it is generated.

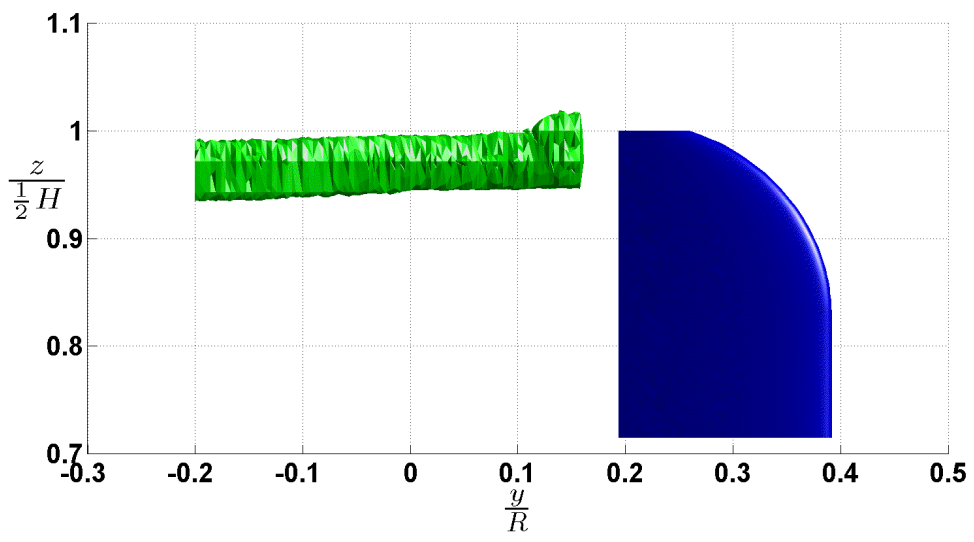

(a) View a).

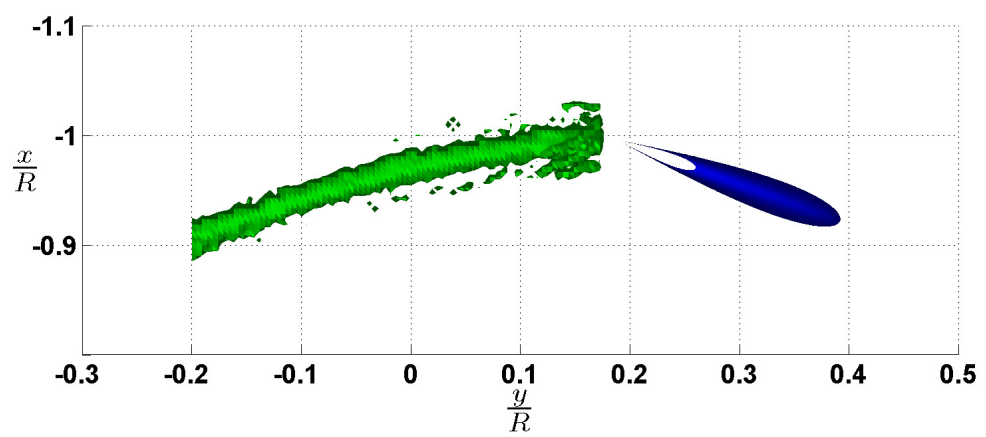

(b) View b).

Figure 4: $3 D$ reconstruction of the vorticity iso-surface $\left(\frac{\Omega c}{U_{\infty}}=30\right)$, at $\theta=110^{\circ}$.

Figures 5 and 6 show the equivalent iso-surfaces for $\theta=150^{\circ}$. The plot of the vortex core between $-0.2<y / R<0.7$ clearly shows the inboard motion and the curvature in the $x$-direction. Although difficult to perceive from these results, there is an azimuthal variation of the inboard motion of the tip vortex, as well as a variation in the convection in the $x$-direction. We will use the PIV results on the planes slicing the tip vortex (the $x z$ planes along the $y$-direction) to analyze the variation with azimuth angle of the tip-vortex convection velocity in the $x$ - and $y$-directions (Sections D and IV).

\section{D. $2 D$ tip-vortex slices in $x z$-planes}

Figures 7 to 10 show the $2 D$ vorticity distribution on the slices of the tip vortex generated by the straight trailing edge blade tip $T E$, on the planes $\frac{y}{R}=-0.42,-0.21,0,0.21$ and 0.42 , for azimuth angles of the rotor $\theta=80^{\circ}$ to $190^{\circ}$. The figures illustrate the convection and evolution (the vortex core expansion) of the tip vortex at different planes in the upwind half of the rotor.

The plots show:

- The wake shed from the blade tip, composed of the tip vortex and the wake released from the remainder of the tip, which is rolled into the tip vortex and dissipated.

- The inboard $\left(\frac{2 z}{H}<1\right)$ motion of the tip vortex in regions close to $y / R=0$.

- The expansion of the tip-vortex core. 


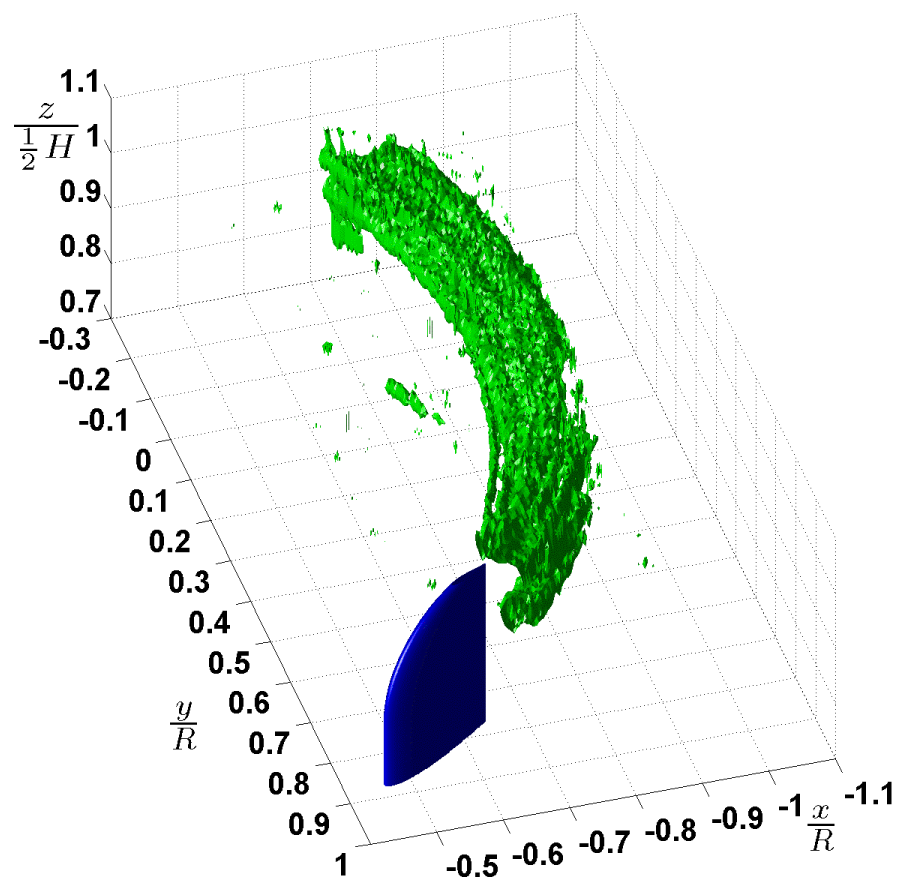

(a) View a).

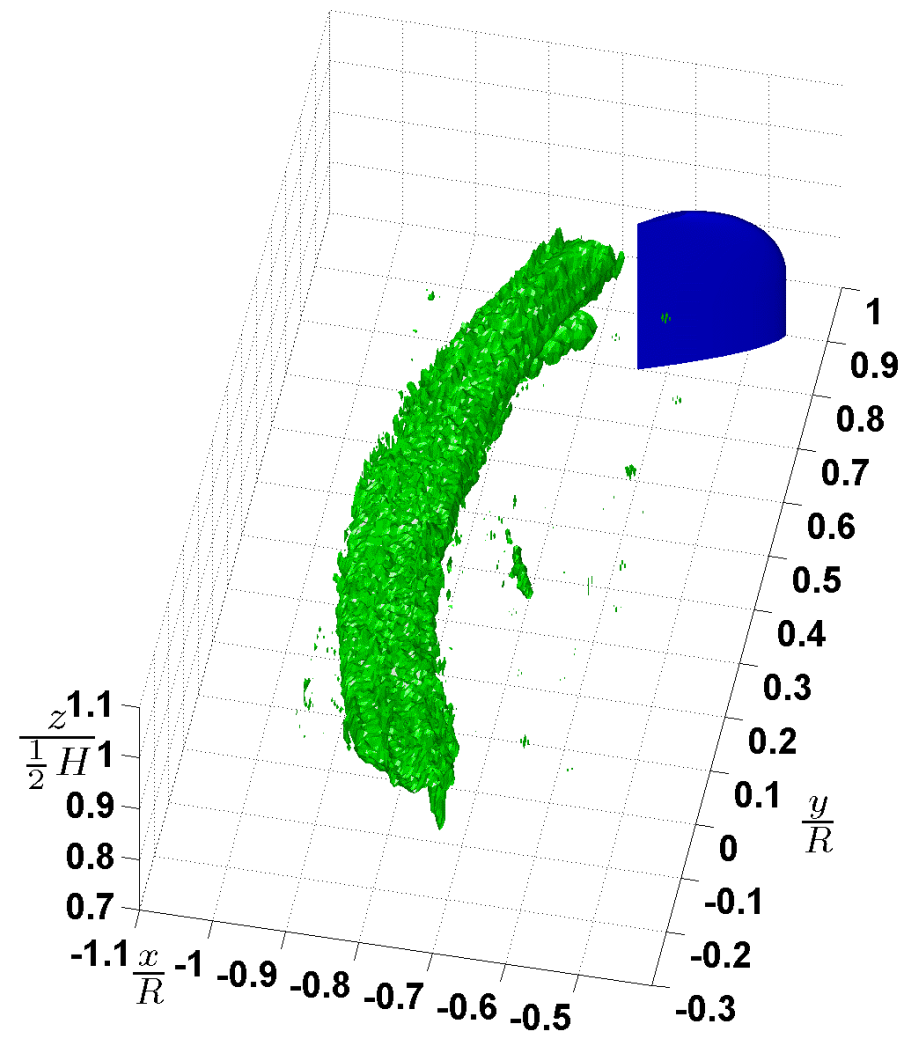

(b) View b).

Figure 5: $3 D$ reconstruction of the vorticity iso-surface $\left(\frac{\Omega c}{U_{\infty}}=5\right)$, at $\theta=150^{\circ}$. 


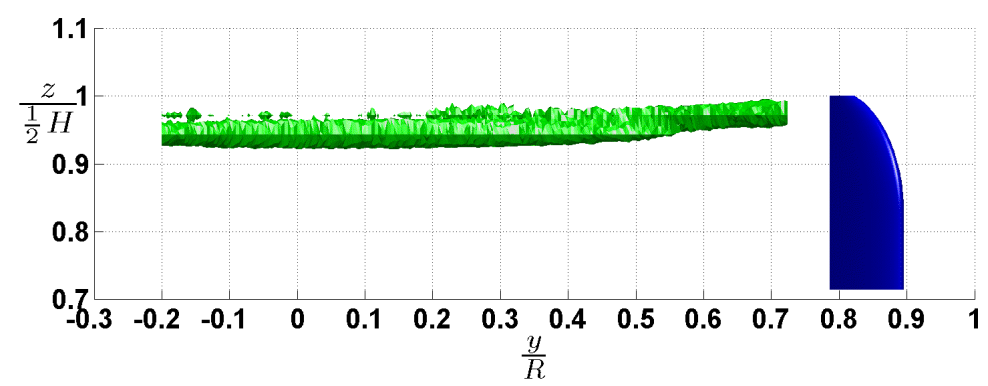

(a) View a).

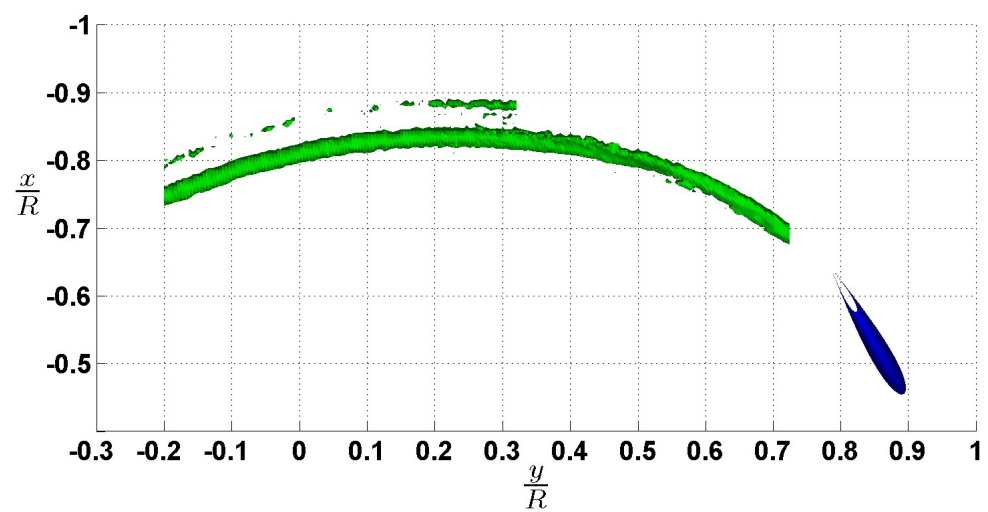

(b) View b).

Figure 6: $3 D$ reconstruction of the vorticity iso-surface $\left(\frac{\Omega c}{U_{\infty}}=30\right)$, at $\theta=150^{\circ}$.

\section{Tip-vortex convection}

Figures 11 and 12 present the location of the intersection of the tip-vortex core with the measurement planes at $y / R=-0.63,-0.42,-0.21,0 ., 0.21,0.42$, and $y / R=0.63$.

The estimation of the location of the tip-vortex core is performed by a weighted average of the vorticity of the tip-vortex flow; despite the lower particle density at the vortex core, and thus, the higher uncertainty on the value of vorticity at the core, the estimation of the location of the vortex core is not significantly affected (see the work of ${ }^{9}$ ).

An alternative method of estimating the location of the vortex core (and the circulation) is to assume a vortex model and adjust/optimize the model to match the observed tangential velocity field (see, for example ${ }^{10}$ ). This method adds two challenges: first, it requires a correct choice of vortex model; second, it requires the $2 D$ velocity data plane to be perpendicular to the $3 D$ vortex core. From Figure $2 \mathrm{~b}$, it is possible to see that the current measurement planes are, with the exception of the case $y / R=0$, not perpendicular to the vortex core line.

\section{A. Trajectory in the $z$-direction}

Figure 11 shows the trajectory of the intersection between the tip-vortex core and the measurement planes, viewed in the $y$-direction.

The evolution in the $z$-direction of the trajectory of the tip vortex clearly varies along the $y$-direction. For most of the values of $\|y\|$, the tip vortex clearly moves inboard $\left(z_{\text {tip }}<0\right)^{\mathrm{a}}$; however, in the windward region of the blade passage (for example, $y / R=-0.63)$, the tip vortex clearly has an outboard $\left(z_{t i p}>0\right)$ motion.

\footnotetext{
${ }^{\mathrm{a}} z_{\text {tip }}$ represents a local coordinate system where the $z=0$ is taken as the blade tip height.
} 


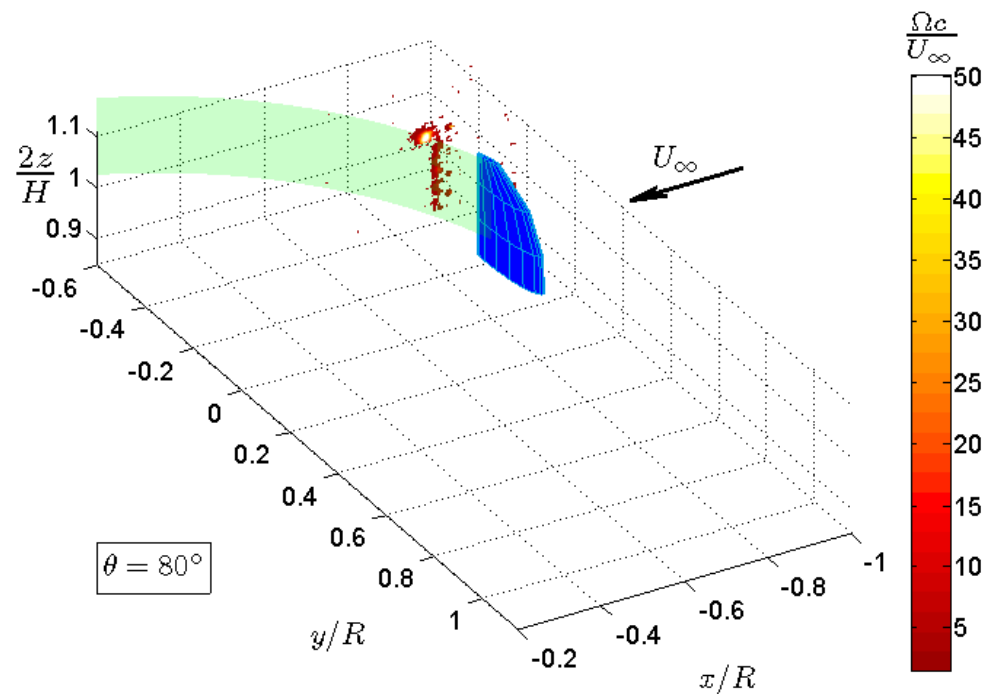

(a) $\theta=80^{\circ}$.

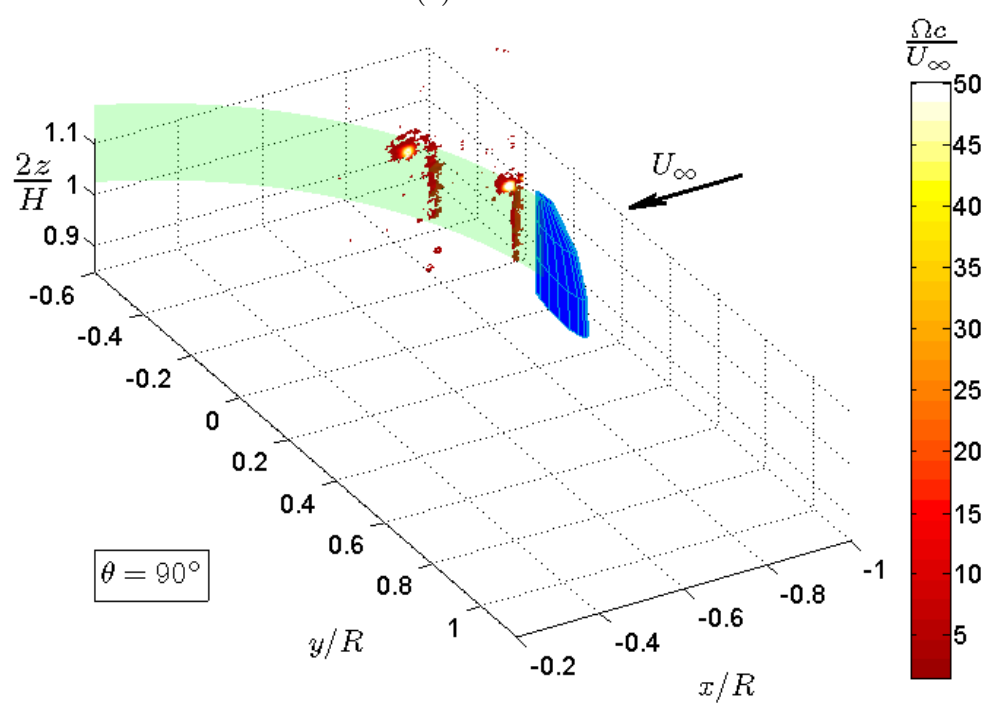

(b) $\theta=90^{\circ}$.

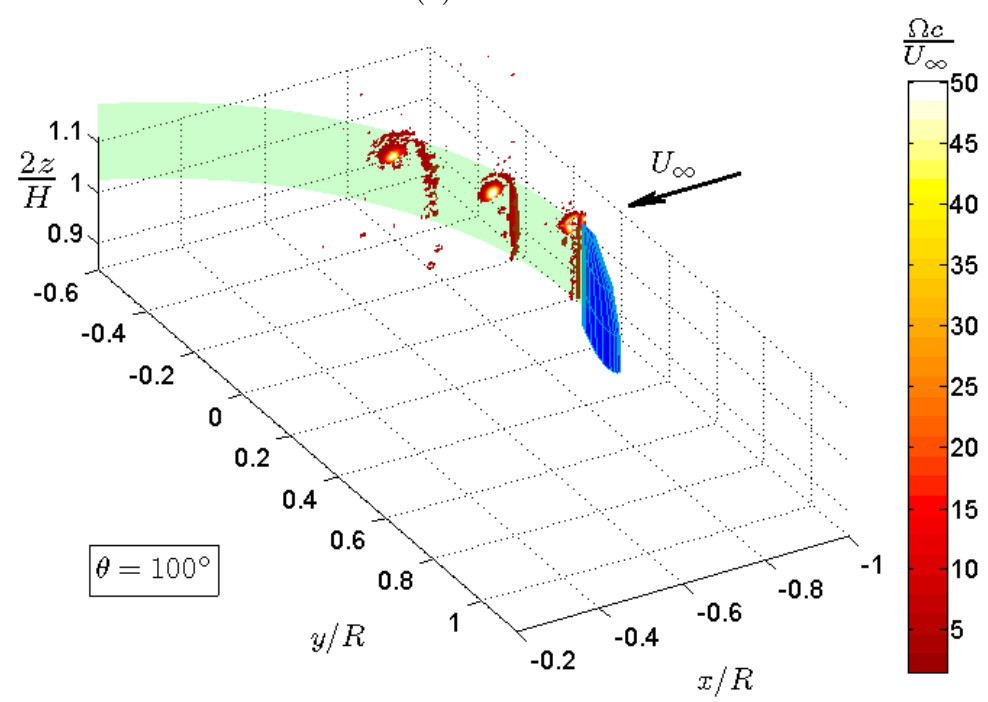

(c) $\theta=100^{\circ}$.

Figure 7: Experimental PIV measurement of vorticity on planes $\frac{y}{R}=-0.42,-0.21,0$ and 0.21 , for azimuth angle of the rotor $\theta=80^{\circ}, 90^{\circ}$, and $100^{\circ}$. 


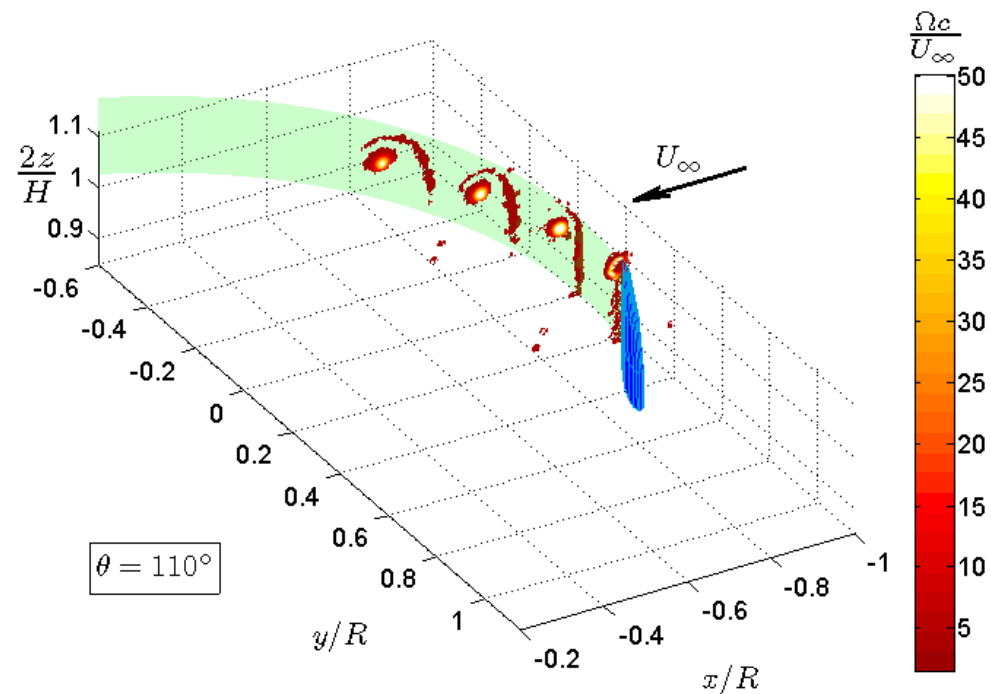

(a) $\theta=110^{\circ}$.

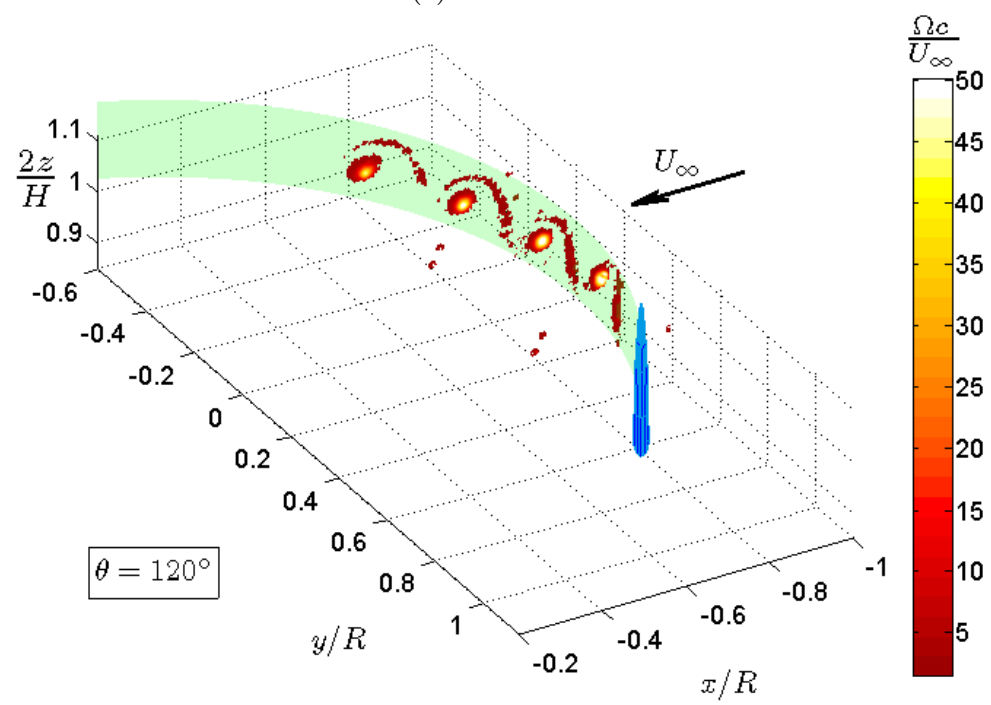

(b) $\theta=120^{\circ}$.

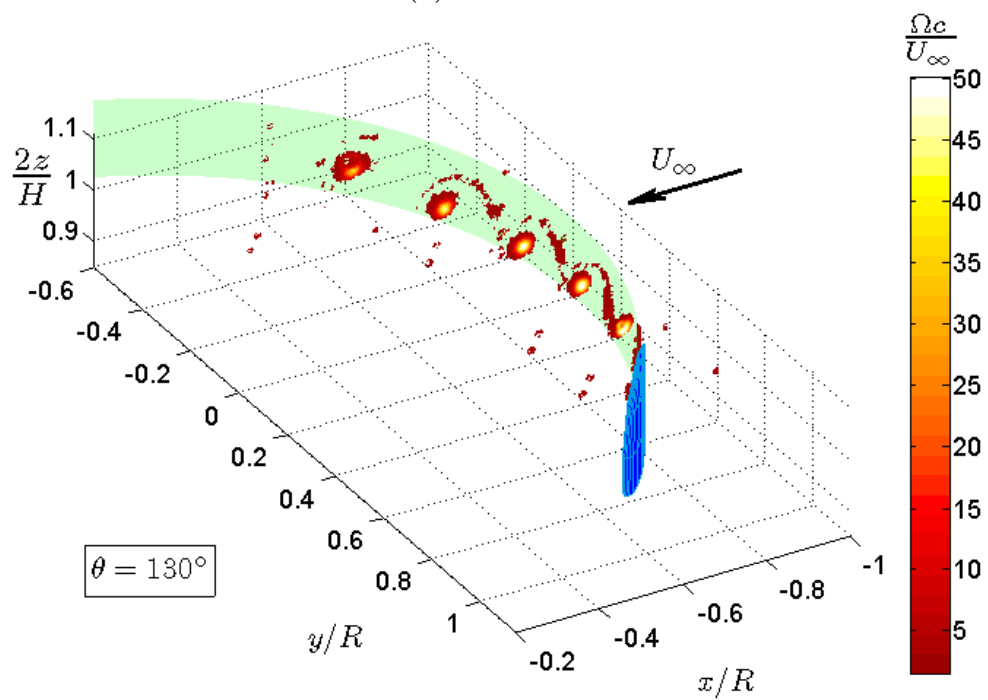

(c) $\theta=130^{\circ}$.

Figure 8: Experimental PIV measurement of vorticity in planes $\frac{y}{R}=-0.42,-0.21,0,0.21$ and 0.42 , for azimuth angle of the rotor $\theta=110^{\circ}, 120^{\circ}$ and $130^{\circ}$.

10 of 33 


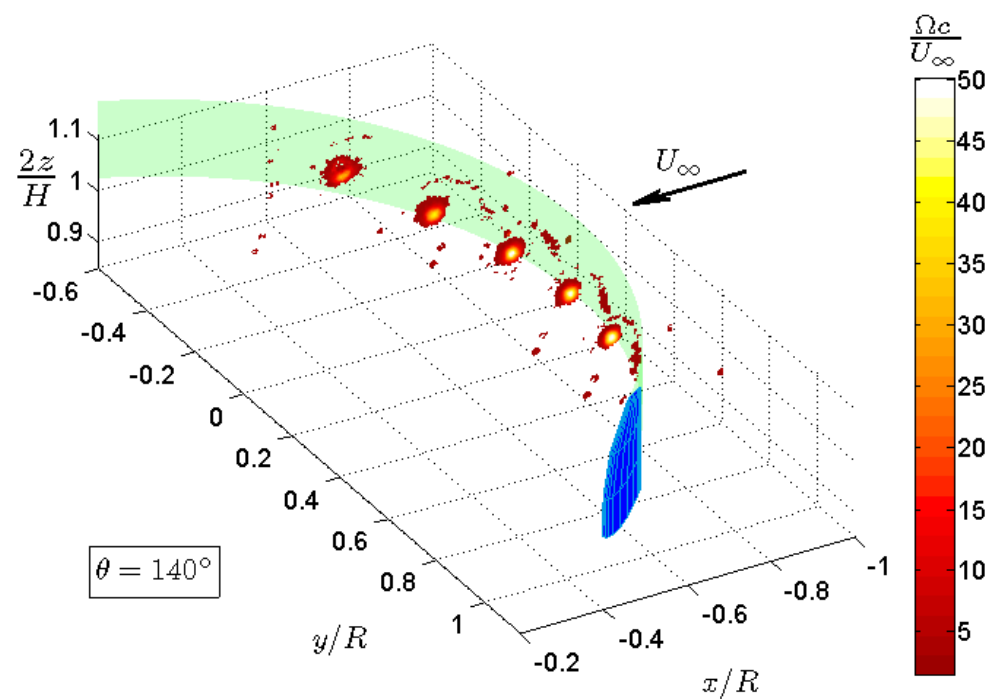

(a) $\theta=140^{\circ}$

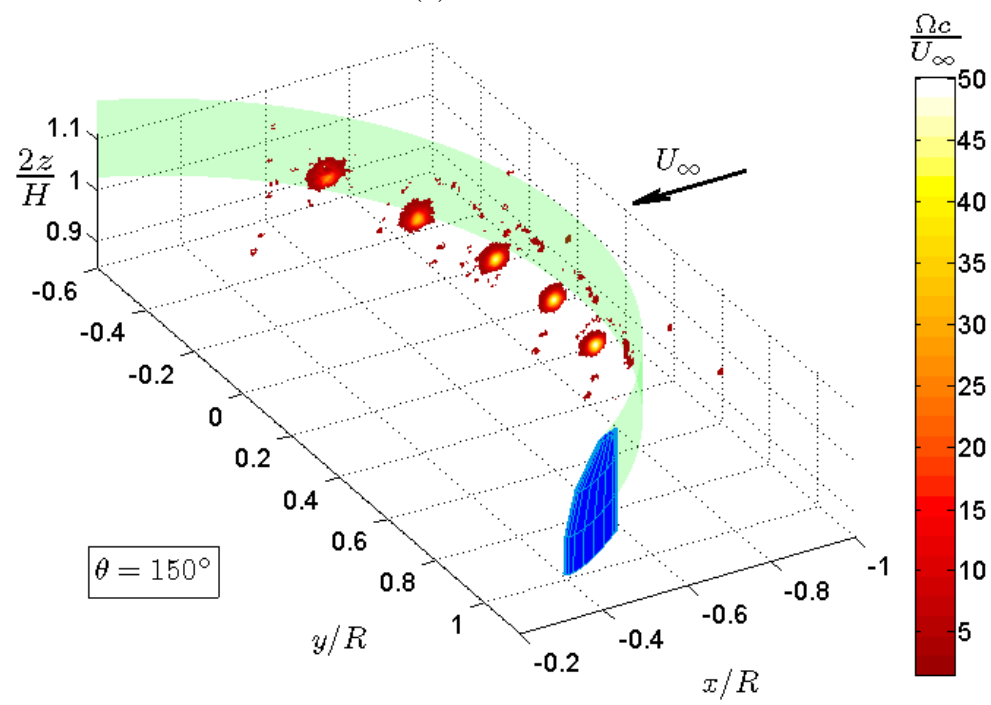

(b) $\theta=150^{\circ}$

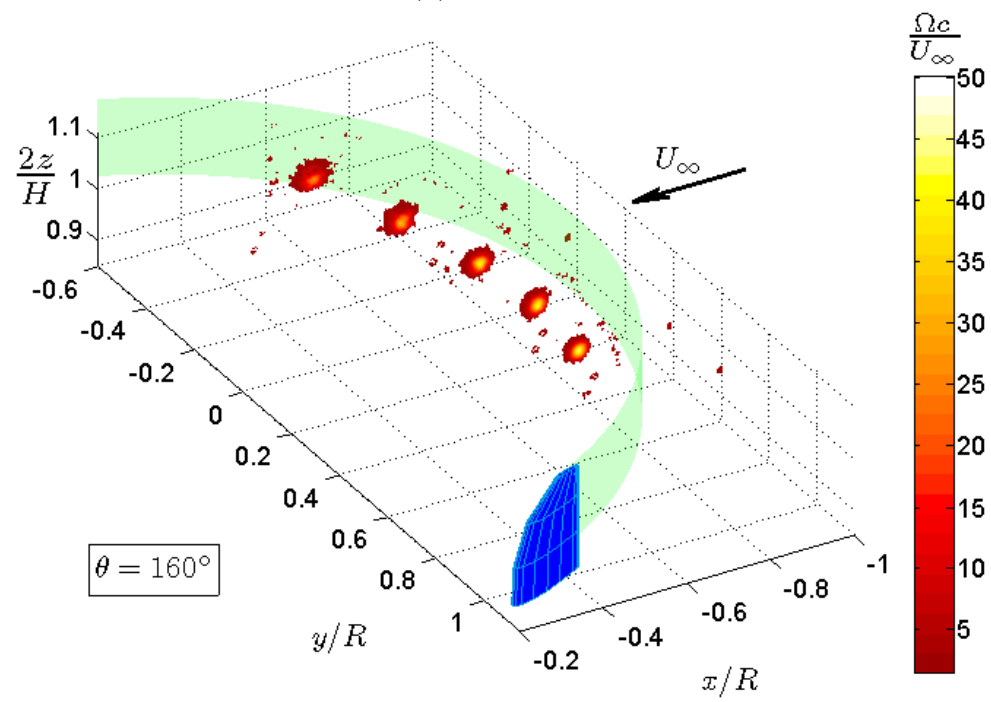

(c) $\theta=160^{\circ}$.

Figure 9: Experimental PIV measurement of vorticity on planes $\frac{y}{R}=-0.42,-0.21,0,0.21$ and 0.42 , for azimuth angle of the rotor $\theta=140^{\circ}, 150^{\circ}$ and $160^{\circ}$.

11 of 33 


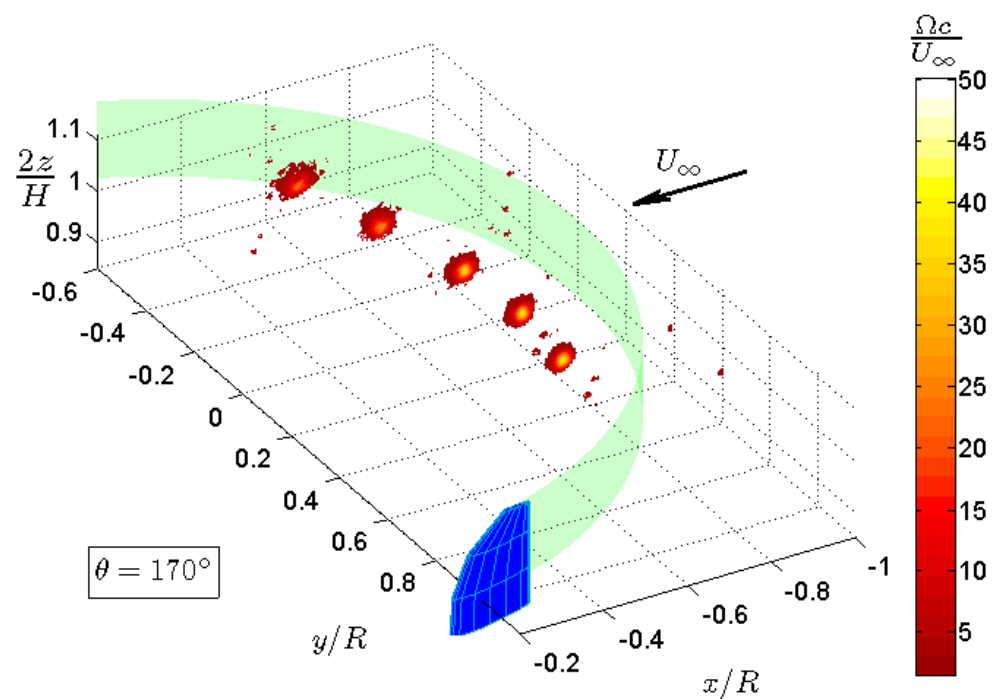

(a) $\theta=170^{\circ}$.

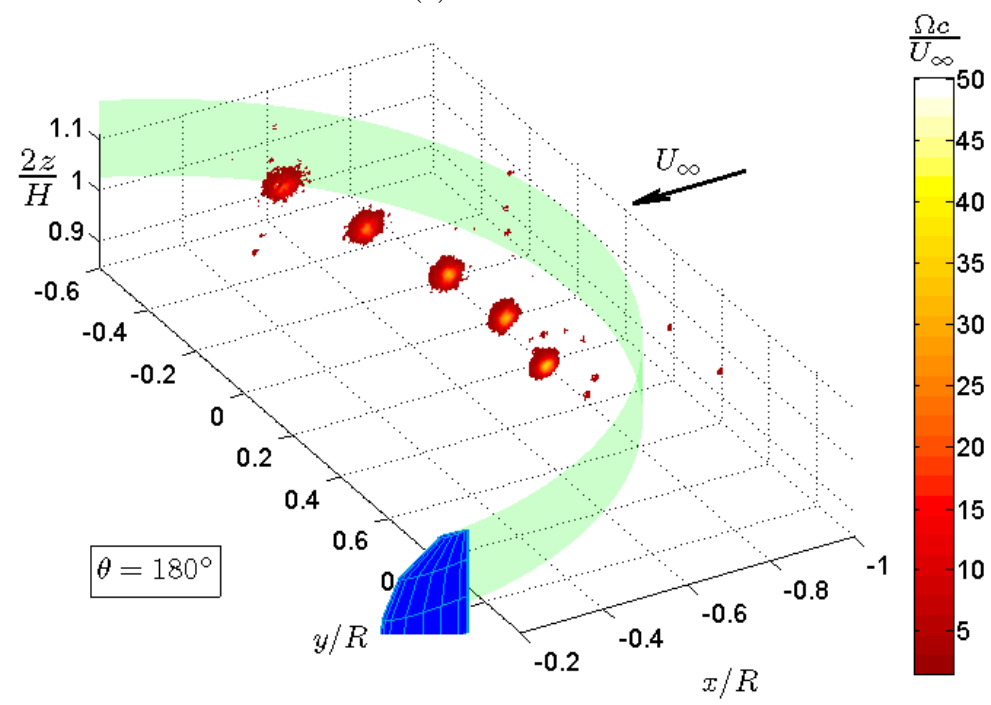

(b) $\theta=180^{\circ}$

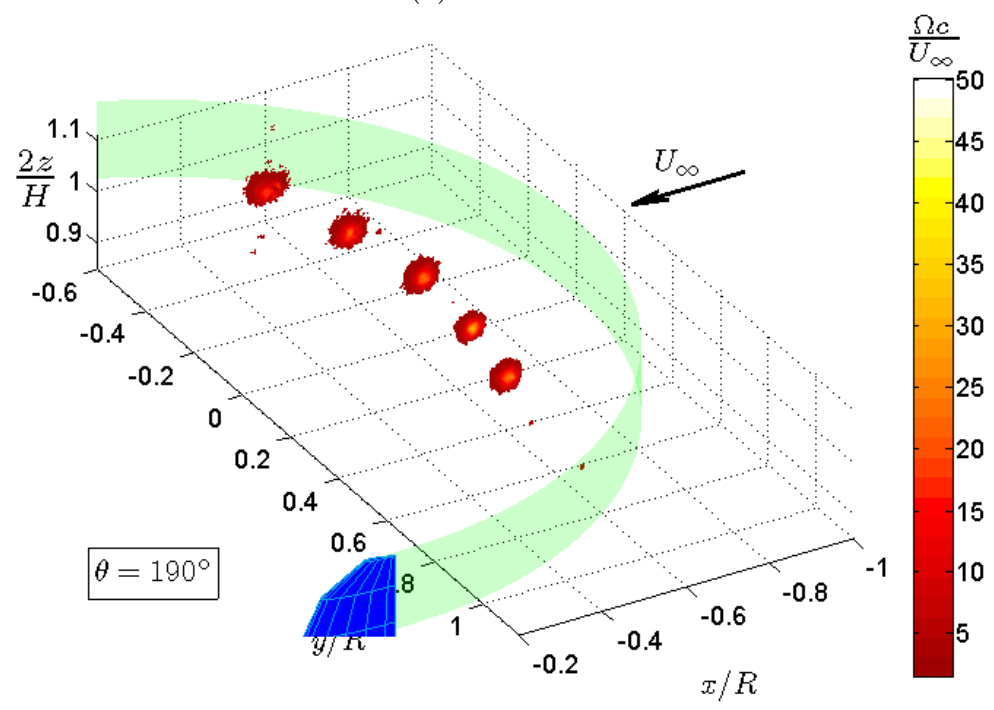

(c) $\theta=190^{\circ}$.

Figure 10: Experimental PIV measurement of vorticity on planes $\frac{y}{R}=-0.42,-0.21,0,0.21$ and 0.42 , for azimuth angle of the rotor $\theta=170^{\circ}, 180^{\circ}$ and $190^{\circ}$.

12 of 33 


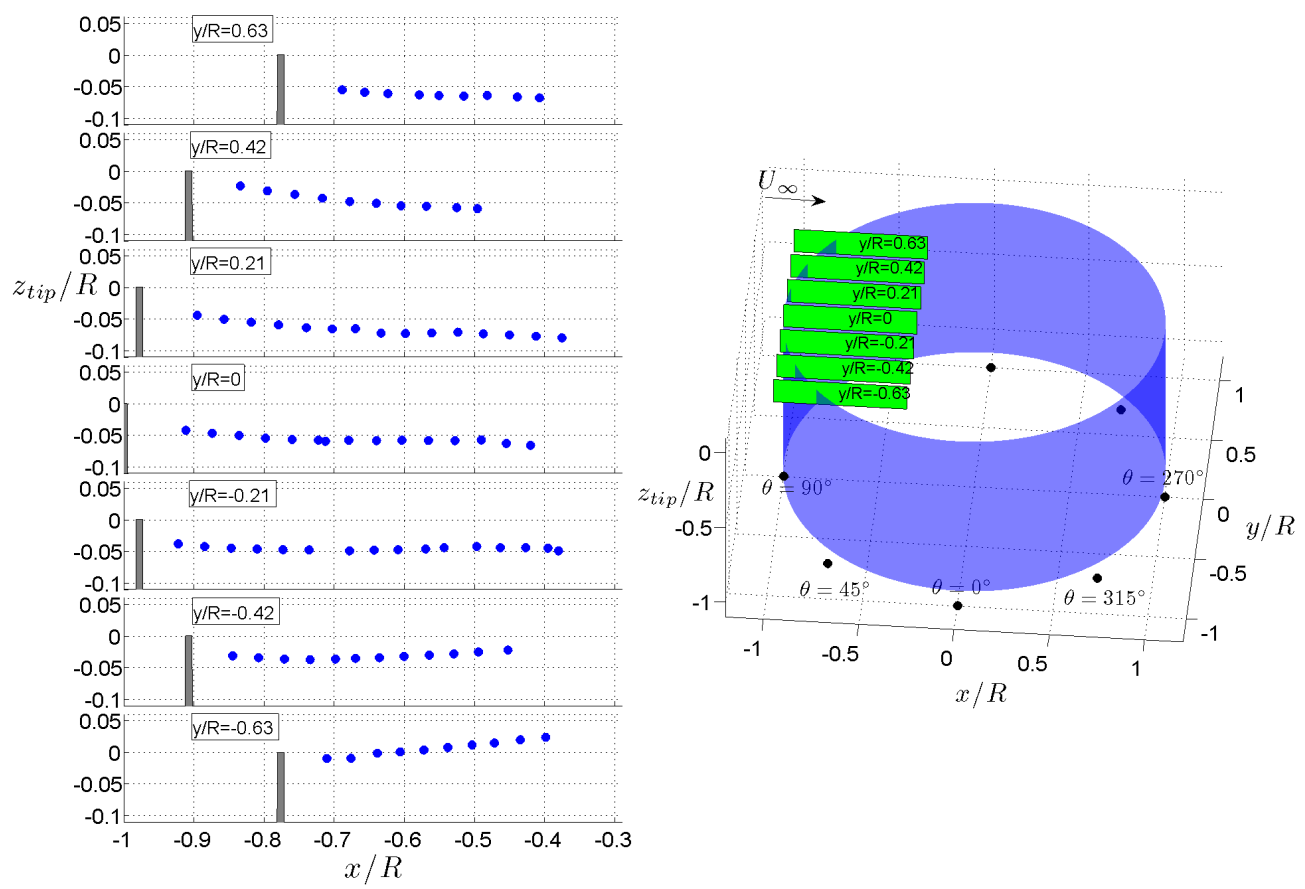

Figure 11: Location of the tip-vortex core at different planes, and schematic of the planes.

\section{B. Induction in the $x$-direction}

Figure 12 shows the intersection between the tip-vortex core and the measurement planes for several azimuthal positions, view from the negative $z$-direction. For comparison, the geometric position (with no induction included) of the tip-vortex core for each azimuthal position is plotted.

The comparative plot clearly shows the effect of induction in the $x$-direction on the trajectory of the tip vortex. In Figures 3 and 5, a small portion of the wake sheet rolling over the tip vortex is visible, corresponding to the part of the wake sheet located at $\frac{2 z}{H}>0.68$. In this region, the tip vortex is further downwind than the remainder of the wake vorticity, indicating that the tip vortex experiences the lowest induction in the $x$-direction.

\section{Evolution of tip-vortex strength}

The strength/circulation of the tip vortex is one of the most challenging parameters to measure; it requires the definition of what is the limit contour of the tip vortex. The tip vortex is just one component of the trailing vorticity sheet, so the calculation of the tip-vortex strength implies a definition of a boundary between what constitutes the tip vortex and the remaining wake.

One way of overcoming this is to apply the methodology mentioned in Section IV, using a vortex model to estimate the circulation from curve fitting the velocity distribution. Notice that this method does not eliminate the effect of the presence of the tip vorticity on the remaining wake, and it also presents the previously-mentioned challenge of overcoming the non-perpendicularity between the tip-vortex core and the measurement plane.

For this experimental data set, the circulation is integrated in the region bounded by an arbitrary isovorticity contour (a low absolute value of vorticity), and an arbitrary cut-off between the tip vortex and the remaining wake. The reader is, once again, referred to the work of ${ }^{9}$ for details on the estimation of the tip-vortex strength.

This method of estimating circulation implicitly forces an underestimation of the total circulation of the tip vortex by not using the null vorticity contour as a boundary. It also presents a challenge imposed by the dynamics of the formation of the tip vortex; the newly-released vorticity at the blade tip tends to 


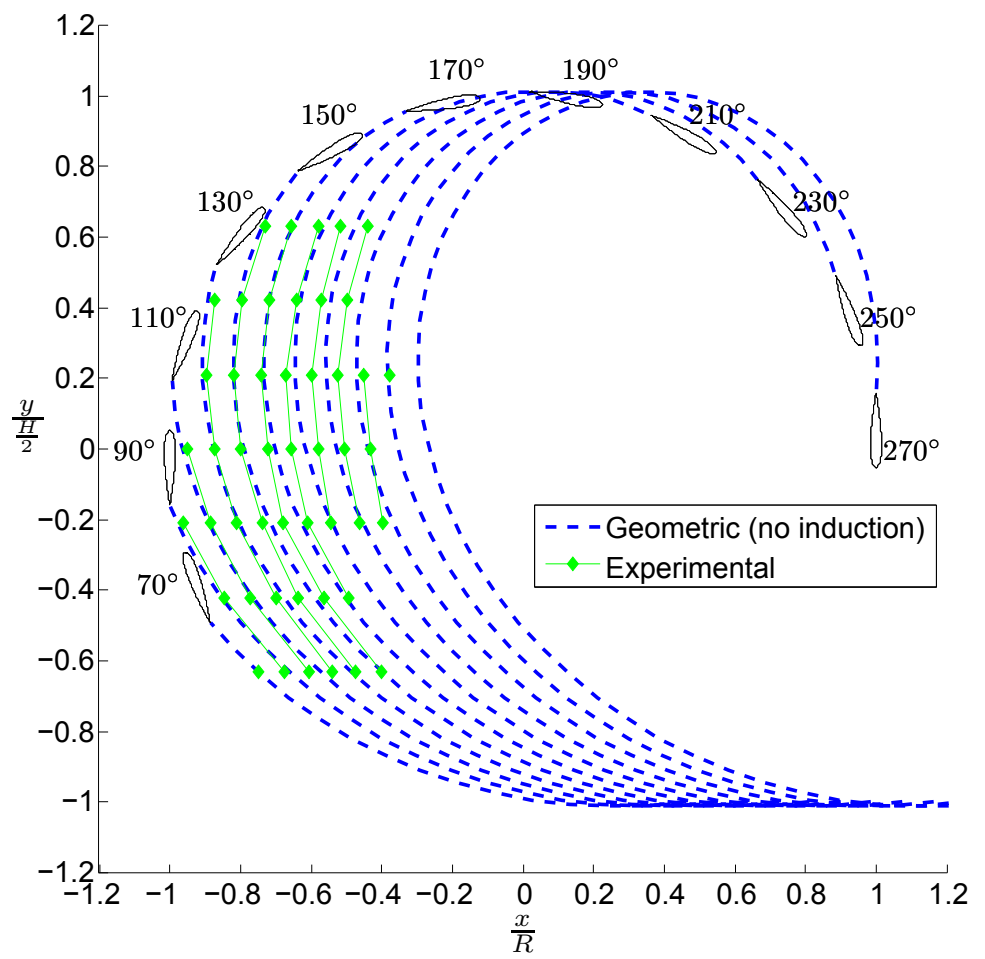

Figure 12: Location of the tip-vortex core ( $x y$ view) in the different measurement planes for several azimuth angles; comparison with fixed geometric wake location (no induction).

roll-up, increasing the strength of the tip vortex at the same time that dissipation of blade-tip vorticity is occurring. Thus, there is an uncertainty associated with the moment at which the tip vortex can be considered completely formed.

The last source of uncertainty/bias is the previously mentioned misalignment between the tip-vortex core and the normal to the measurement planes. For small angles, the impact of this misalignment on the calculation of circulation can be approximated by a factor of $\sqrt{\cos }$ of the misalignment angle.

Figure 13 shows the strength of the tip vortex measured at each plane (the location is defined by the azimuthal position of the intersection of the planes with the blade path). The values presented are nondimensionalized by the circulation of the tip vortex at $y=0\left(\theta=90^{\circ}\right)$.

This scaling of the values is based on two reasons:

- The previously-discussed complexity in determining the strength of the tip vortex, and the arbitrary way in which the integration contours are defined, limit the suitability of the absolute values for model validation.

- The implementation of vortex/panel codes makes it relatively difficult to validate the models with the absolute strength of the tip vortex; however, the relative value is a very useful term for validation.

The uncertainty bars of the experimental points do not represent the experimental uncertainty, but instead represent the uncertainty in estimating the average value of the tip-vortex strength over the first $50^{\circ} / \omega$ period of rotation past the generation of the tip vortex at each plane location.

\section{The $3 D$ free-wake panel model of the VAWT}

The simulations of the $3 D$ VAWT flow are performed using the $3 D$ free-wake panel model developed by ${ }^{10}$ (see also ${ }^{11}$ ). The VAWT modeled is the same as that used for the experimental study.

The 3D, unsteady, multi-body, free-wake panel method is intended as a design and research tool to capture the $3 D$ nature of a VAWT and its wake. The model contains methods to realistically treat blade-wake 


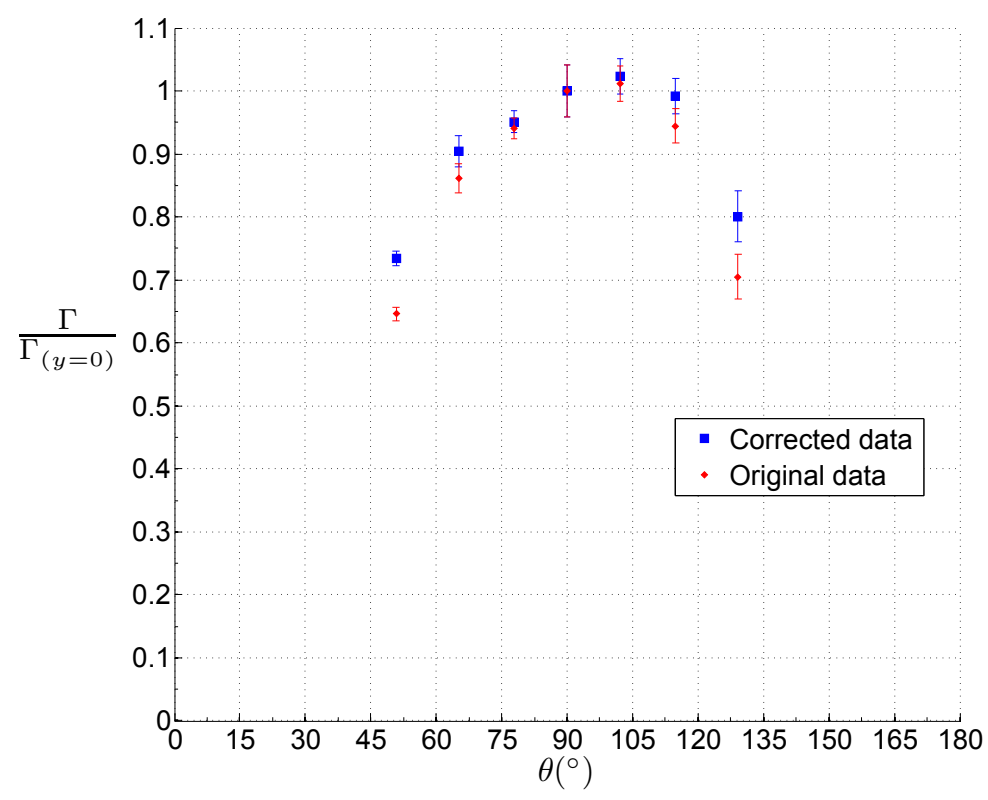

Figure 13: Tip-vortex strength over azimuth, non-dimensioned by tip-vortex segment at $y / R=0$; measured data non-corrected and corrected for misalignment angle between vortex core and the measurement plane.

interactions, vortex stretching/contraction and viscous diffusion. Validation of the model is demonstrated by $^{10}$ and $^{11}$ by comparison with PIV data and with smoke trail studies for straight-bladed VAWTs.

The panel model uses potential flow elements to model the blades, wakes and incoming flow field. The blades are modeled with $3 D$ surface panels of sources and doublets with a constant distribution. The near wake is modeled with a distribution of doublets, while the far-wake is modeled with a mesh of vortex rings. The wake is modeled with self-induction (including blade) and free convection, and the wake vortex elements are implemented with a vortex core and stretching model.

The non-entry boundary condition on the aerofoil surface is implemented by imposing a Dirichlet boundary condition on the potential at the surface. Additional details of the model implementation can be found in the thesis of. ${ }^{10}$

\section{Development and convection of the tip vortex generated upwind}

Figure 14 shows a view of the surface swept by the convection of the tip vortex generated during the upwind passage $\left(0^{\circ}<\theta<180^{\circ}\right)$ (simulated data); Figures 15 and 16 show additional perspectives of the surface. The color of the surface varies with the original $z$-coordinate of the wake element, and the lines over the surface indicate the position of the tip vortex tube at several azimuthal positions.

In this section, we focus on the tip vortex segment generated during the upwind passage $\left(0^{\circ}<\theta<180^{\circ}\right)$ in order to understand the impact of $3 D$ effects of the near wake, in particular, the induction due to trailing vorticity and the interaction of the upwind-generated tip vortex with the blade during the downwind passage.

The surfaces in Figure 14, which represent the loci of the upwind-generated tip vortex as it convects downwind, show an effect of the distribution in the $y$-direction of the induction in the $x$-direction. The largest induction occurs closer to the axis of rotation $\|y / R\|<0.5$; the tip vortex segment is convected downstream faster at locations of larger $\|y / R\|$.

Figure 15 shows the front and back views of the surfaces swept by the tip vortices. These views show an important event in the convection of the tip vortex: the inboard movement $(\|2 z / H\|<1)$ of the tip vortex for locations $y / R>-0.5$, and the outboard movement $(\|2 z / H\|>1)$ of the tip vortex at regions $y / R<-0.5$. This asymmetry in the $y$-direction of the inboard/outboard movement is related to the blade motion and blade-wake interaction; the tip vortex in the region close to the blade during its windward motion $(y / R<0)$ has a outboard motion, while during the leeward motion, although there is a small motion in the outboard direction, the tip vortex wake sheet tends to roll-up inboard. We will discuss in detail the source 


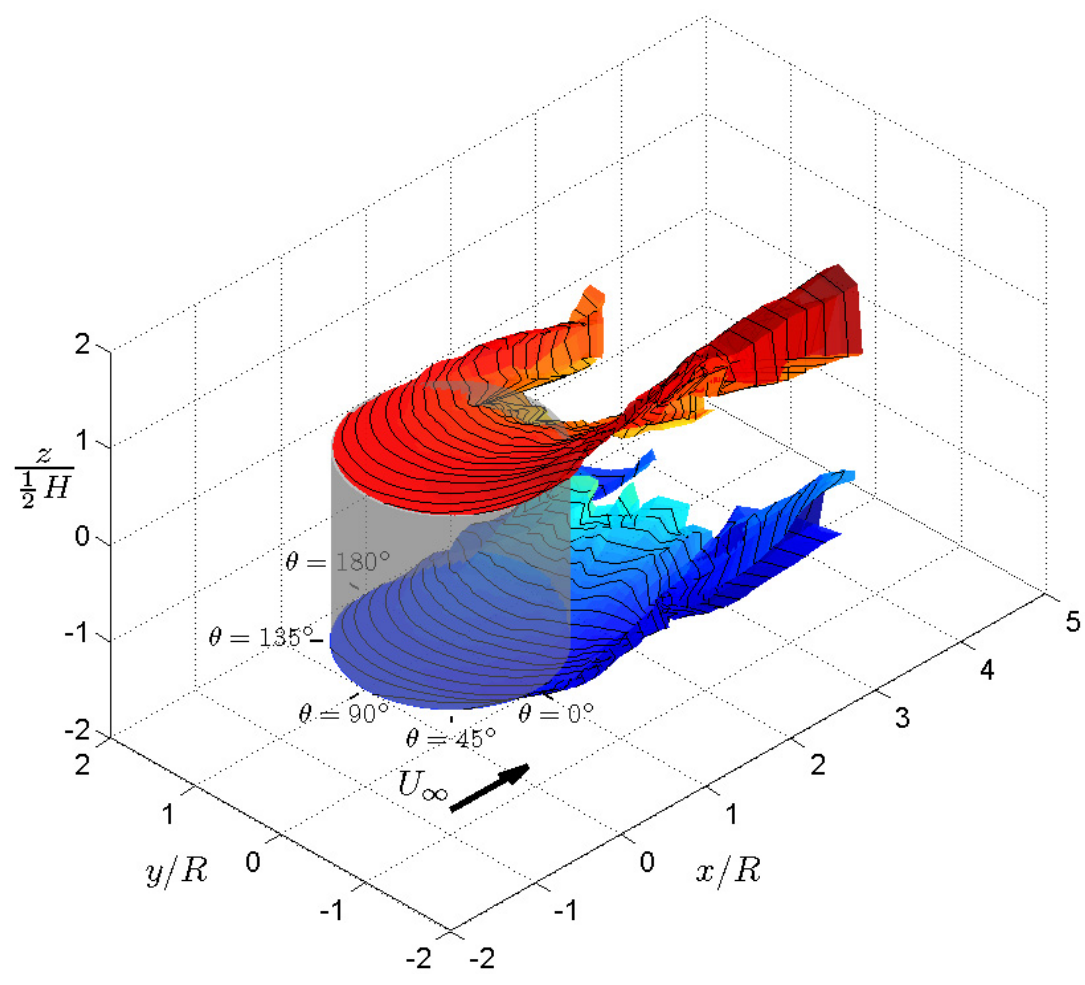

Figure 14: Angle view of the surface described by the convection of the tip vortex filament released in $0^{\circ}<\theta<180^{\circ}$.

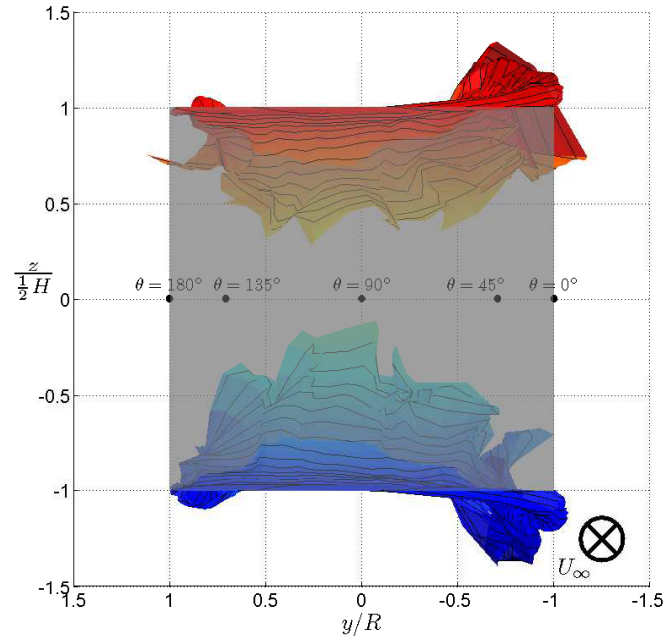

(a) Front view.

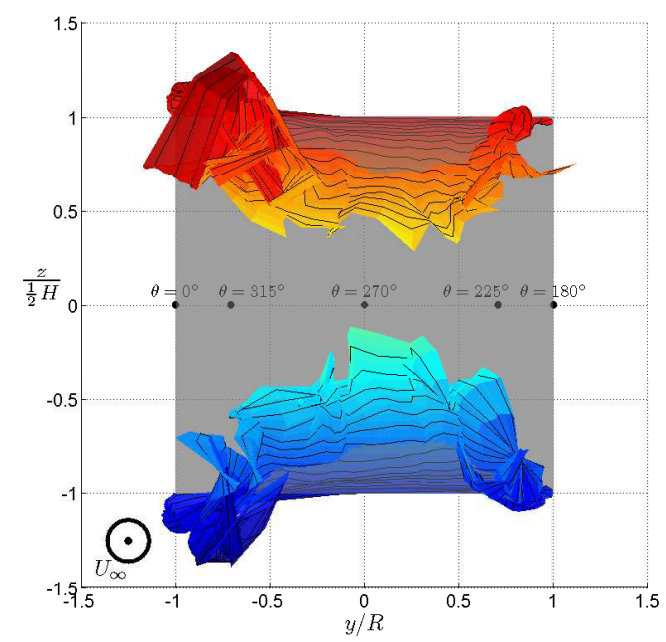

(b) Back view.

Figure 15: Front and back views of the surface described by the convection of the tip vortex filament released in $0^{\circ}<\theta<180^{\circ}$. 
of this difference in Section IX.

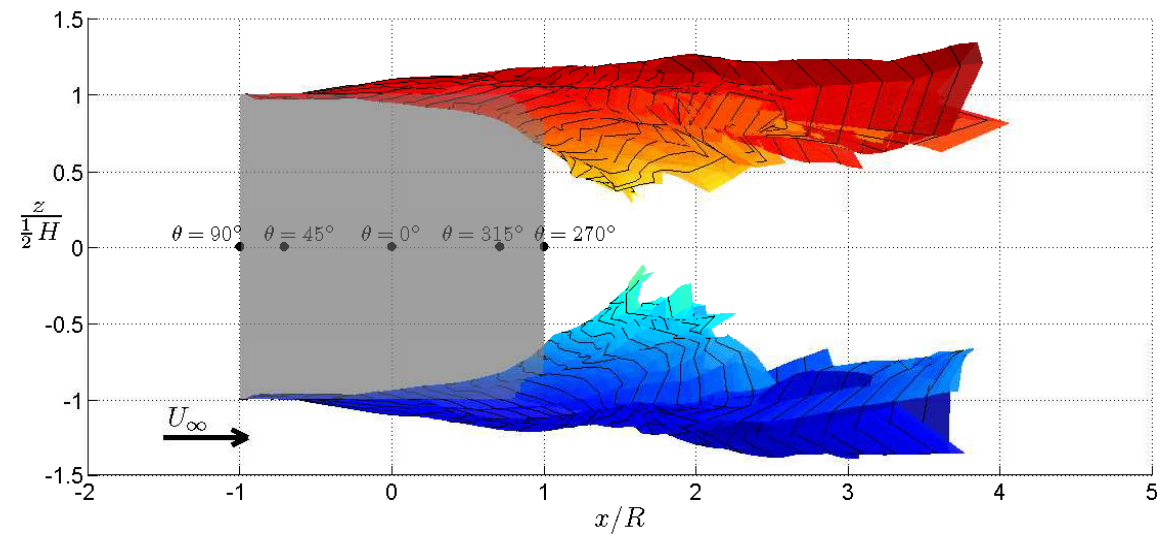

(a) Side view.

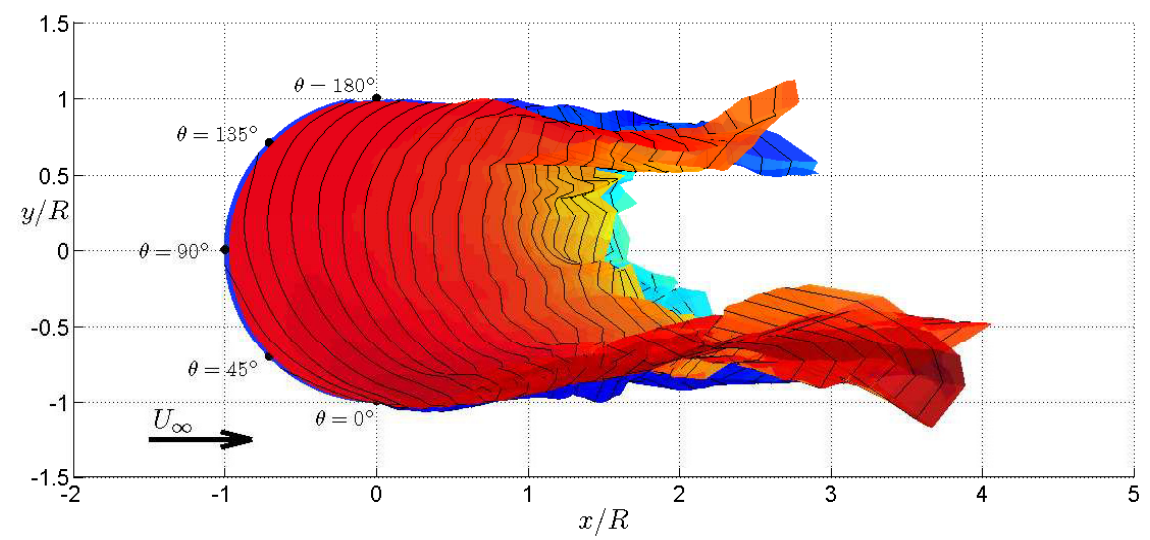

(b) Top view.

Figure 16: Side and top views of the surface described by the convection of the tip vortex filament released in $0^{\circ}<\theta<180^{\circ}$.

The inboard motion of the tip vortex and the resulting interaction with the downwind blade passage is also visible in Figure 16a. The downwind blade passage is then divided in three regions, delimited by the location of the tip vortex swept surface. The induction of the strong tip vortex generated upwind will create a large variation of induction over the blade during the downwind blade passage; we will discuss this in more detail in Section X.

The top view presented in Figure 16b shows the effect of the roll-up of the vortex sheet in the $y$-direction; the tip vortex rolls up at the regions of leeward and windward motion of the blade, moving into the rotor diameter region $(\|y / R\|<1)$.

\section{Validation with experimental results}

In this section we validate the simulations with the experimental results presented.

\section{A. Location of the tip vortex}

Figure 17 shows a plot of the iso-vorticity $\left(\frac{\Omega c}{U_{\infty}}=5\right)$ surface calculated from the experimental PIV data and the wake calculated by the panel code, both at $\theta=150^{\circ}$. The simulated wake grid and the experimental data are in good agreement in terms of location of the roll-up region of the tip vortex. However, the application in the simulation of a first-order integration model for the convection of the wake implies some limitations on tracking the location of the tip vortex core. To better see this, we will examine the results in more detail, using Figure 18. 


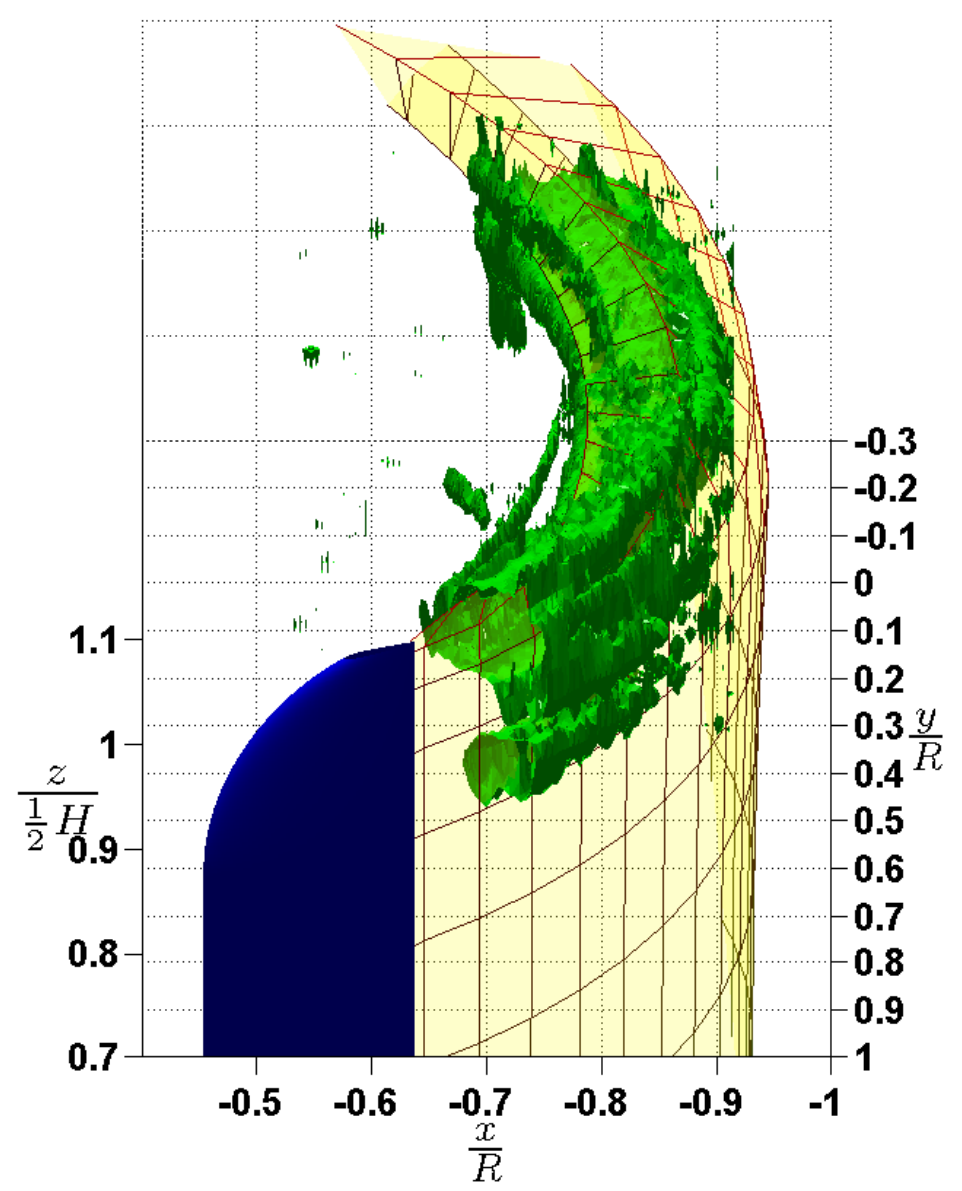

Figure 17: Comparison of the $3 D$ reconstruction of experimental iso-vorticity surface $\left(\frac{\Omega c}{U_{\infty}}=5\right)$ and the near wake from the panel model, at $\theta=150^{\circ}$. 
Figure 18 shows three orthogonal views of the iso-vorticity $\left(\frac{\Omega c}{U_{\infty}}=30\right)$ surface calculated from the experimental PIV data and the wake calculated by the panel code, at $\theta=150^{\circ}$. The three views show that, although the position of the vortex core in the $x y$ plane is well predicted by the simulation, the model seems to underestimate the inboard motion of the tip vortex in the $y z$ plane.

The source of the difference in performance of the model for these two motions relates to the physics upon which the models are based. The induction that drives the motion in the $x y$ plane can be split into two parts:

- The induction of the several wake sheet segments generated during previous rotations; the model is able, with the level of time and space discretization used in the simulation, to account for the induction due to the trailing and shed vorticity of the wake sheets from other blades and at other positions in the of rotation.

- The near-field induction of the roll-up motion of the tip vortex, which explains the curvature in the $x z$ plane of the wake sheet close to the tip.

Since the convection of the wake is dominated by the free stream velocity and by the first of the induction terms above mentioned, the model shows a good agreement with experiments in the $x y$ plane, as seen in Figures $18 \mathrm{a}$ and 19.

However, the effect of the first-order model for the wake convection, and the coarse discretization of the wake, make the model less accurate in capturing the second induction term; this can be seen in Figure 18c, where the detail (in the $x z$ plane) of the curvature of the wake sheet close to the tip is visible.

The difficulty in discretizing the roll-up motion finely enough affects the accuracy of the motion in the $y z$ plane; in this plane, the motion of the tip vortex core (in the region here observed) is dominated by the roll-up motion of the tip vortex, which is heavily affected by the teh resolution of the discretization of the model.

Figure 20 shows a comparison between the trajectory of the tip vortices at several $y$ planes obtained from PIV data and simulated with the $3 D$ free-wake panel code.

The coarseness of the grid, both in space and time step, coupled with the first order linear Newton method used to calculate the convection of the wake, causes the model not to capture the precise roll-up trajectory of the tip vortex, probably due to its inability to accurately capture the roll-up and entrainment of the trailing vorticity at the tip. However, when comparing the effect initial tip vortex location in the $y$-direction, both experimental and simulation data present the same trend.

The model can capture the impact of the location in the $y$-direction on the inboard/outboard motion of the tip vortex, previously identified by PIV measurements.

The model can also capture the main trends of curvature in the $z$-direction of the tip vortex. However, further improvements in the roll-up model and higher order integration methods need to be implemented to capture the correct tip vortex roll-up.

\section{B. Tip vortex strength}

In the $3 D$ unsteady free-wake panel model, the trailing vorticity sheet is discretized into doublets and vortex filaments. The experimental results for the tip vortex strength consider a boundary contour, arbitrarily defined, for which the trailing vorticity contained inside the contour is considered as part of the tip vortex, and the remaining trailing vorticity is considered as part of the remaining wake sheet.

Thus, when trying to validate the simulation results against experimental values, the largest challenge is to define the region of trailing vorticity that is considered as part of the trailing vortex and as part of the remaining wake sheet. Any choice, based on the available data, is arbitrary. For example, for the simulation here presented, with the current discretization, considering the last three or four trailing vortices filaments at the blade tip as corresponding to the tip vortex, the resulting value of circulation varies $\pm 6 \%$ in relation to the experimental value

However, the definition of the boundary contour used for integration of circulation using the experimental data was consistent throughout the analysis of experimental data for the strength of the tip vortices at the different $y / R$-planes. Using also a consistent measurement of the strength of the tip vortex obtained from the simulation (for example, the total circulation of the three trailing vortex filaments at the tip), we can compare the relative variation of tip vortex strength with azimuth. 


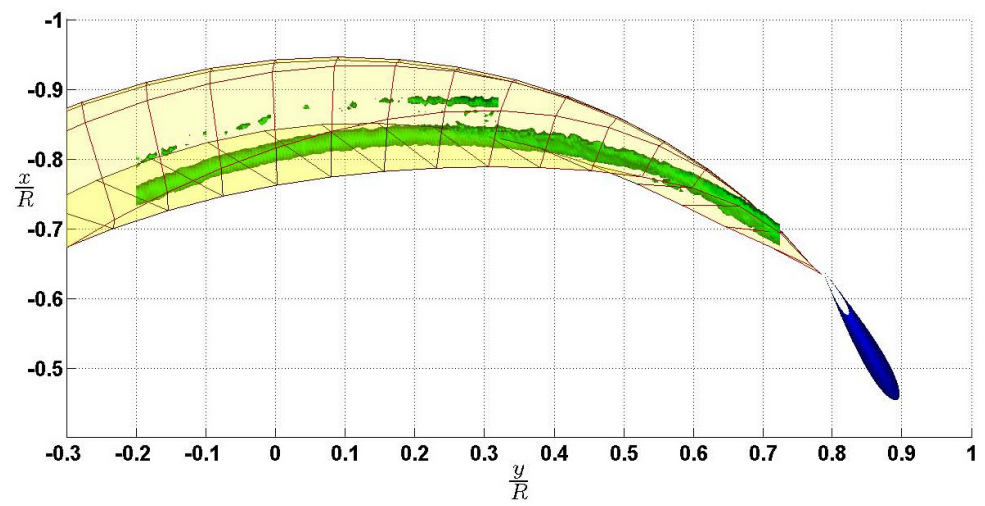

(a) View a), $x y$ plane.

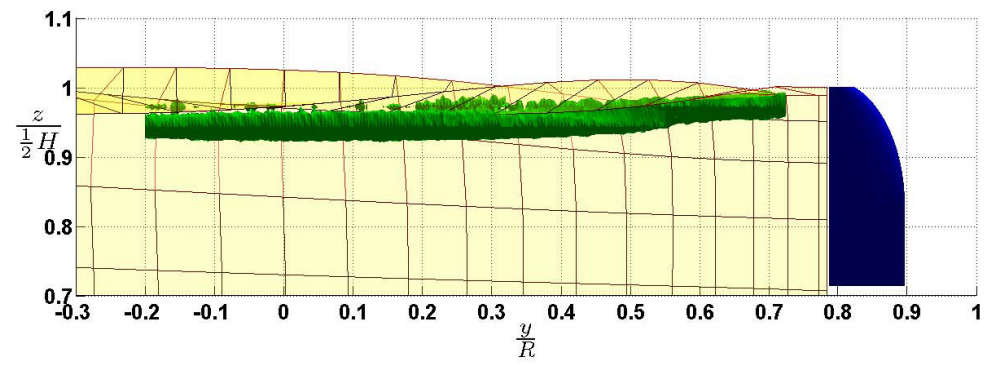

(b) View b), $y z$ plane.

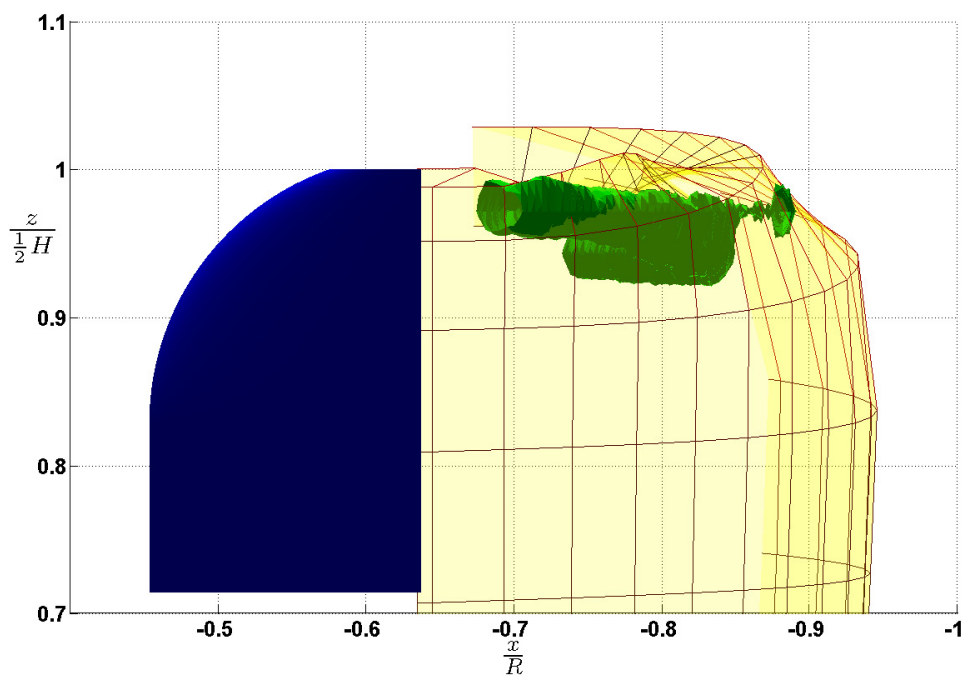

(c) View c), $x z$ plane.

Figure 18: Comparison of the $3 D$ reconstruction of experimental iso-vorticity surface $\left(\frac{\Omega c}{U_{\infty}}=30\right)$ and the near wake from the panel model, at $\theta=150^{\circ}$. 


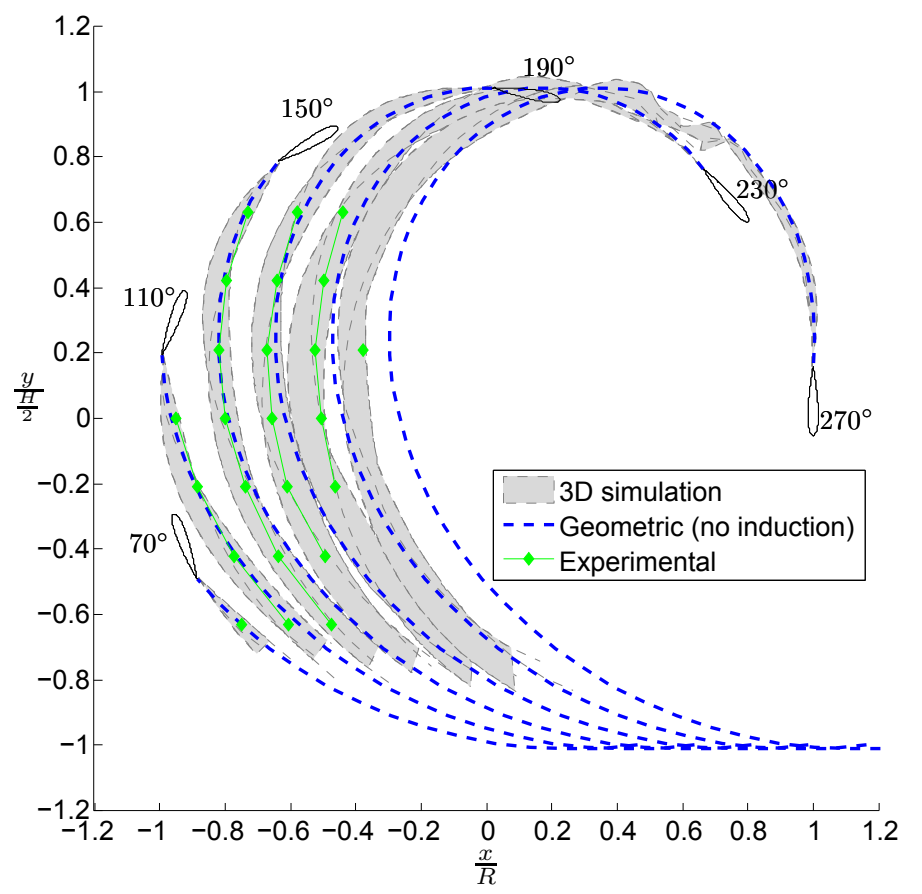

Figure 19: Comparison of the location of the tip vortex ( $x y$ view); simulation results with $3 D$ free-wake panel code, fixed geometric wake (no induction) and experimental data from PIV measurements.

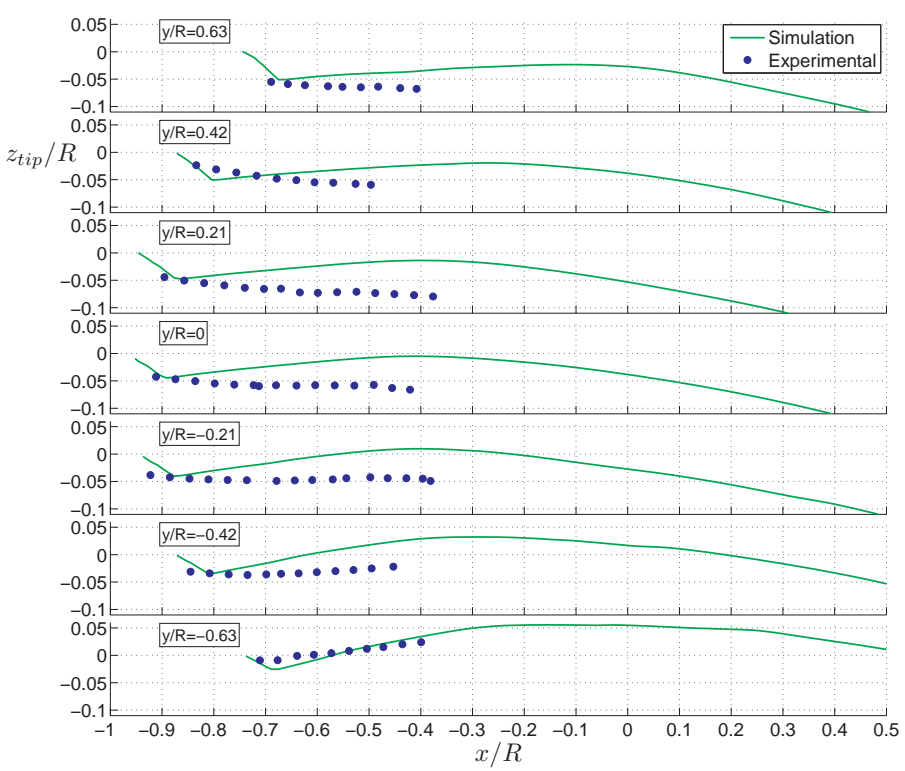

Figure 20: Position of vortex core in the measurement planes ( $y$-view). 
Figure 21 shows a comparison between simulation and experiments for the value of circulation of the tip vortex; the values are scaled by the value of circulation of the segment of tip vortex generated when the blade is at $\theta=90^{\circ}$, both for the experimental and simulation data.

The experimental results are corrected to compensate for the angle between the plane of measurement of circulation and the direction of the vortex tube, which is approximated by a $\cos ^{\frac{1}{2}}$ relation.

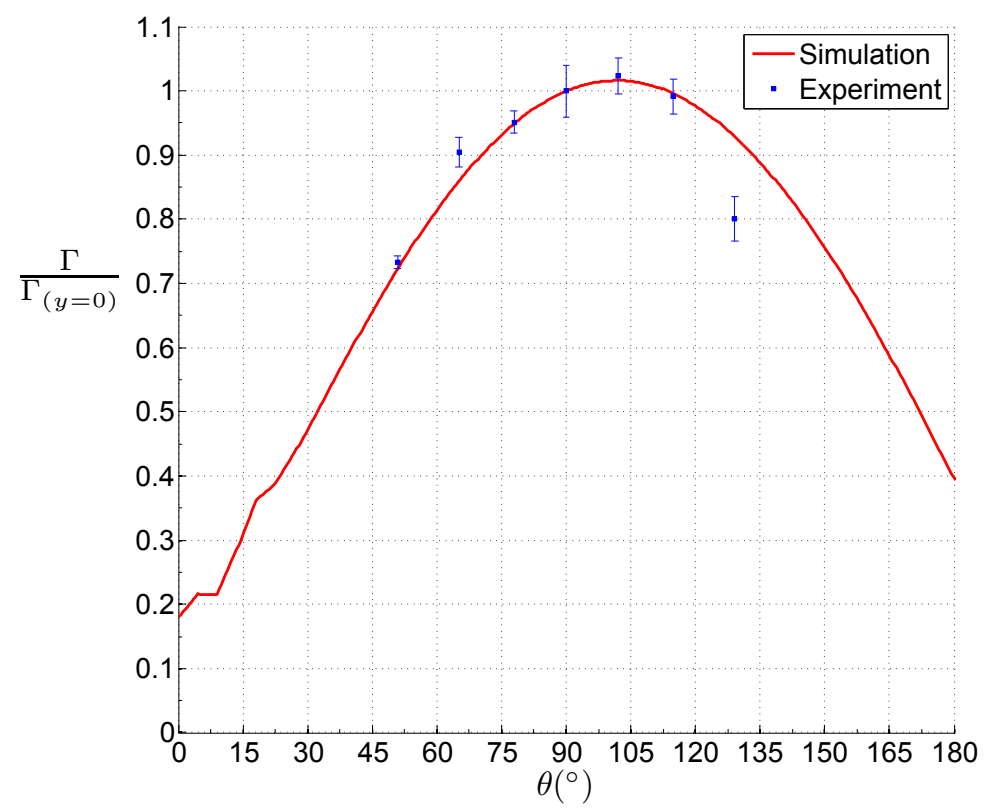

Figure 21: Relation between experimental values of tip vortex strength and simulated panel model values.

The results in Figure 21 show good agreement of the relative circulation distribution along the tip vortex tube; the only exception occurs at the more leeward measured point. This outlier may be a result of lower quality of the experimental data or a different spanwise distribution of the actual trailing vorticity.

\section{Asymmetry in the $y$-direction of the wake sheet expansion and roll-up}

In the previous section we observed the asymmetry in wake roll-up and inboard/outboard movement of the wake between the leeward and windward regions or rotation. Although the flow is asymmetric in the $y$-direction already in the $2 D$ case, the spanwise $z$-direction adds an additional effect of asymmetry. In this section we will explore the mechanism behind this flow feature.

Figure 22 plots the simulated rotor and wake at $\theta=90^{\circ}$. The plot shows two aspects of the asymmetry

- In the windward region of the wake, the expansion at midspan $(z=0)$ is stronger that in the leeward region. This effect was already present in the $2 D$ flow case, since it is mainly driven by the shed vorticity, but it is increased by the trailing vorticity existing in the $3 D$ flow (see Section VIII).

- In the windward region, the expansion of the wake in spanwise (z-direction), and roll-up of the wake close to the tip vortex is larger than in the leeward motion.

The direction of roll-up and magnitude of the tip-vortex is not only dependent on the angle of attack, but also on the added circulation due to the constant pitching motion of the blade. The zero-circulation tip vortex will therefore occur in a region between $\theta=315^{\circ}$ and $\theta=0^{\circ}$, as it transitions from downwind to upwind.

\section{X. $3 D$ wake effects on the downwind blade passage}

During the downwind passage of the VAWT blade, the blade operates in the blade-wake interaction and is heavily influenced by the development of the wake generated during the upwind passage.. 

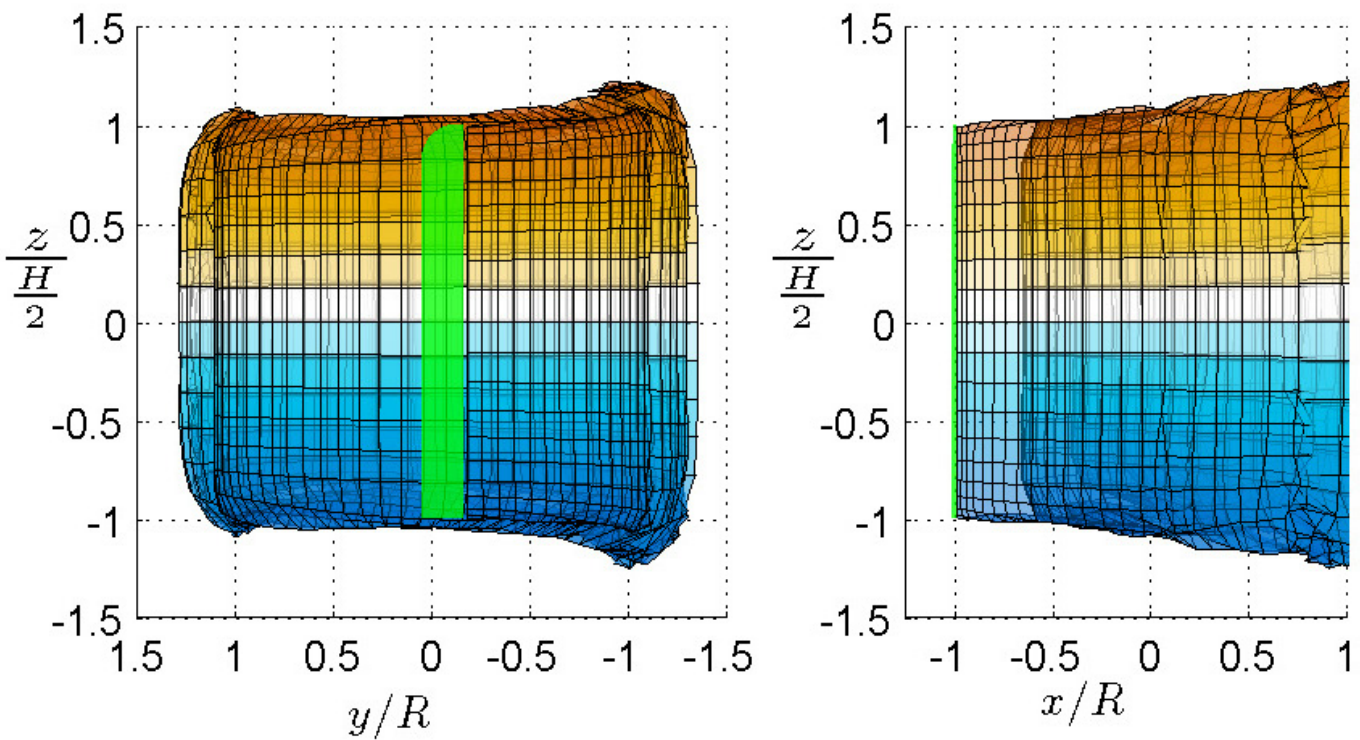

Figure 22: Front and side view of the rotor and wake, $\theta=90^{\circ}$.

Figure 23 shows the azimuthal and spanwise distribution of the different components of vorticity associated with the blade. Figure 23a shows the spanwise distribution of the bound vorticity at each azimuthal position; Figures 23b and 23c show, respectively, the shed and trailing vorticity that is released over the span at each azimuthal position.

The downwind passage bound vorticity distribution can be divided into three regions, determined by the sign of bound vorticity. This variation in bound vorticity results from the location of the intersection between the downwind blade passage and the wake generated during the upwind passage. The influence during the downwind passage of the induction from the upwind generated wake, in particular, that of the tip vortices, can be evaluated in Figure 24. We will analyze first Figure 24, to support the analysis of the results in Figure 23.

The shed vorticity in the downwind blade passage region shows an interesting effect at the midspan region, where the sign of the shed vorticity varies trice, negative-positive-negative-positive. This is a consequence of the large induction at the midspan of the downwind blade passage, where in $3 D$ flow, due to the induction of the tip vortex, the bound circulation experiences a second positive local maximum (see Figure 28). This distribution of shed vorticity represents a loss of efficiency in relation to the $2 D$ flow case.

Figure 24 plots the spanwise induced-velocity distribution at the $1 / 4 c$ position of the blade at three azimuthal positions $\theta=225^{\circ}, 270^{\circ}$ and $315^{\circ}$; the plots are divided into: induced-velocity in the $x$-direction due to shed vorticity in the wake $\left(U_{\operatorname{ind}(x)}\right)$, induced-velocity in the $x$-direction due to trailing vorticity in the wake $\left(U_{i n d(x)}\right)$, and total flow velocity in the $x$-direction $U_{x}$ (resulting from wake induction and $U_{\infty}$ ).

The plots of induction due to shed vorticity clearly indicate the effect of a finite span. The magnitude of the shed vorticity, generated during the upwind blade passage, is lower at the blade-tip region than that at the midspan location; also, due to the tip vortex roll-up, the upwind-generated shed-vorticity (and its induction) is moved more inboard, thus increasing its distance from the blade tip location at the downwind passage.

The induction due to the trailing vorticity in the wake presents the most interesting results. Its order of magnitude is comparable to that of the induction due to shed vorticity, thus clearly causing a decrease of efficiency (in comparison with the infinite span case) of the rotor during the downwind passage. Spanwise, the sense of the induced velocity in the $x$-direction by the trailing vorticity varies; this is caused by the location of the tip vortices of the upwind generated wake, which have moved inboard to positions $\left\|\frac{2 z}{H}\right\|<1$. In the region delimited by the tip vortices, the flow is decelerated, while in the regions outboard of the tip 
vortices, the flow is accelerated.

The plots for the total flow velocity in the $x$-direction in Figure 24 show three regions of the downwind blade passage: a central region of low velocity and two regions, close to the tips, of higher velocity; these regions are characterized by their location between or outboard of the upwind-generated tip vortices, a result of the induction due to trailing vorticity. Contrary to what might be expected, the tip regions experience the largest inflow velocities during the downwind passage.

After understanding the $3 D$ downwind induction field with the help of Figure 24, we can now better understand the vorticity/circulation distribution of Figure 23.

The spanwise bound circulation distribution of Figure 23a shows the impact of the large induction in the blade midspan for $180^{\circ}<\theta<360^{\circ}$. In the midspan region, the circulation due to the flow field is smaller than the circulation due to the movement of the rotor (driven by the pitching motion of the blade). Thus, the value of bound circulation has the same sign as during the upwind passage; this effect is also present in the $2 D$ case as will be shown later in Figure 28. Figure 28 (which will be discussed further in Section B) presents a comparison between the $2 D$ and $3 D$ (midspan location) bound circulation. In the downwind blade passage region $\left(180^{\circ} \theta<360^{\circ}\right)$, the bound circulation in the $2 D$ case is already positive; in the $3 D$ case this effect is even more prominent, due to the larger induction as seen in Figure 24. Figure 23a also shows that, in the tip region, the lower induction causes the circulation due to the inflow to be larger than the bound circulation due to the movement of the blade, resulting in an opposite sign of bound circulation.

The velocity field experienced by the blade during the downwind passage is still dominated by the blade rotation $(\lambda=4$ in this case). Such a velocity field, combined with a sign-varying spanwise bound vorticity distribution, implies that the normal force distribution also varies in sign along the span. In the tip region, the normal force is in the outward direction; however, in the midspan region, the normal force turns inwards (see Figure 25). This force distribution has an effect in the bending and fatigue of the blade. Figure 29 plots a comparison between the $2 D$ and $3 D$ (midspan) normal force along the azimuth; this normal force distribution clearly follows the bound circulation distribution.

Figure $23 \mathrm{c}$ presents four areas of strong release of trailing vorticity for the azimuthal range $180^{\circ}<\theta<$ $360^{\circ}$. These regions are located at the spanwise positions of the blade tips $\left(\frac{z}{H / 2}=1\right.$ and $\left.\frac{z}{H / 2}=-1\right)$ and at the spanwise location near the interaction of the blade with the tip vortices generated during the upwind rotation; this is seen in Figure $23 \mathrm{c}$ as a distribution of released trailing vorticity with a maximum at $\theta \approx 270^{\circ}$ and $\left\|\frac{z}{H / 2}\right\| \approx 0.7$, instead of $\left\|\frac{z}{H / 2}\right\| \approx 1$ has in the upwind region.

\section{Wake at midspan}

The presence of the trailing vorticity in the $3 D$ flow rotor case has an impact on induction, beyond the expected tip vortex region flow.

\section{A. Wake convection velocity}

Figure 26 presents the velocity at the intersection of the wake with the $x$-axis $(z=0, y=0)$, as the wake travels downstream, for the $2 D$ and $3 D$ flow cases. The results show two important effects:

- The $3 D$ flow shows a significant lower velocity than the $2 D$ flow, corresponding to a larger induction.

- The deceleration of the wake is not uniform (it varies in the $x$-direction), resulting in an oscillation of the velocity in the $x$-direction.

The non-uniform deceleration of the wake is a result of:

- The rotation of the blades, which varies the distance and direction between the wake element and the blade bound circulation and blockage effects. As the wake approaches the axis of rotation (see Figure 26, top sub-plot), this varying effect decreases, increasing once again as the wake moves from the axis of rotation towards the downwind passage of the blade.

- The release of new wake, due to both trailing and shed vorticity.

Figure 27 follows on the results of Figure 26 and presents the values of induction (wake induction only) at the wake element as it travels over the $x$-axis line $(y / R=0 z / H=0)$, as calculated by the $2 D$ and the 


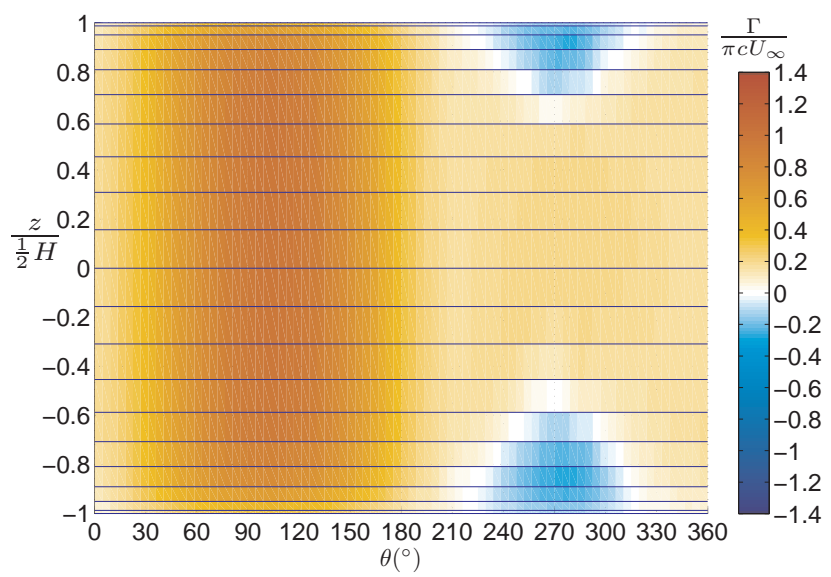

(a) Bound vorticity.

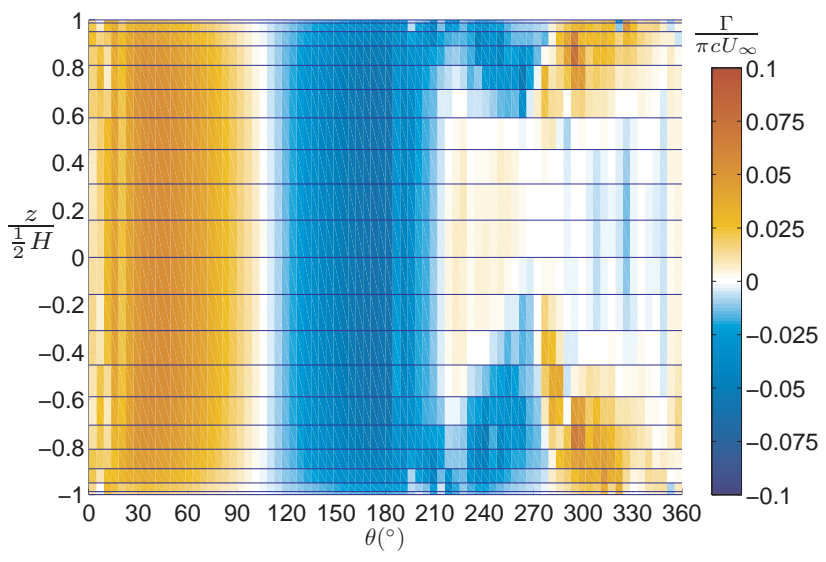

(b) Shed vorticity.

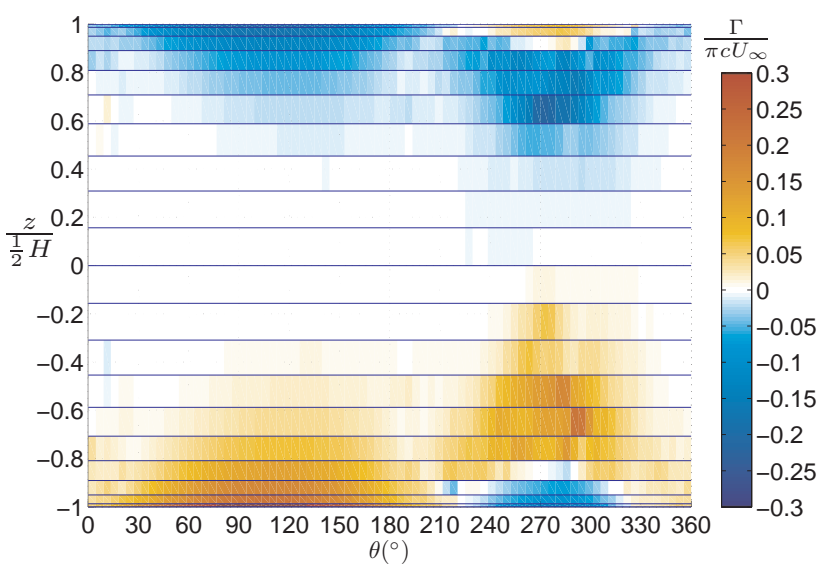

(c) Trailing vorticity.

Figure 23: Vorticity distribution at each azimuthal position, over span: bound, shed and trailing vorticity. 


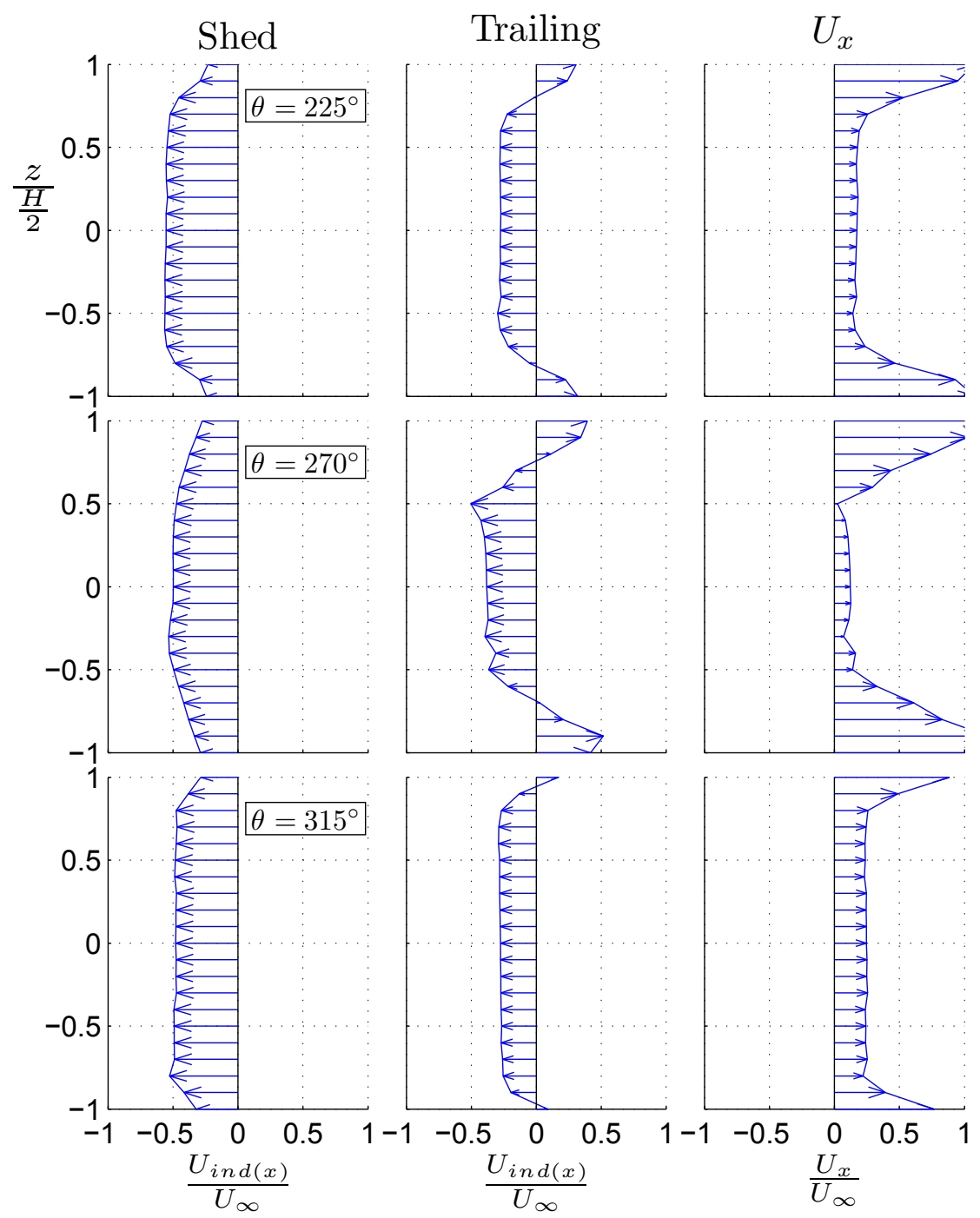

Figure 24: Wake induction velocities over span at three downwind azimuthal positions $\theta=225^{\circ}, 270^{\circ}$ and $315^{\circ}$.

26 of 33 


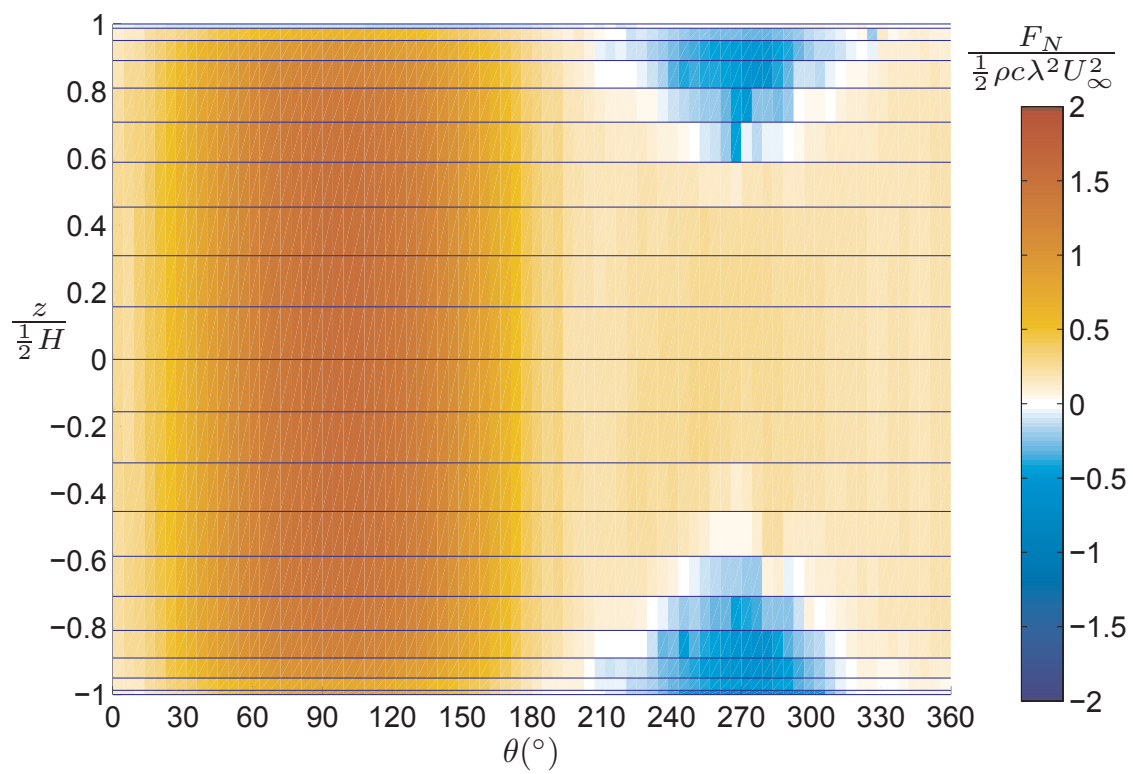

Figure 25: Spanwise distribution of normal force over the blade during the rotation.
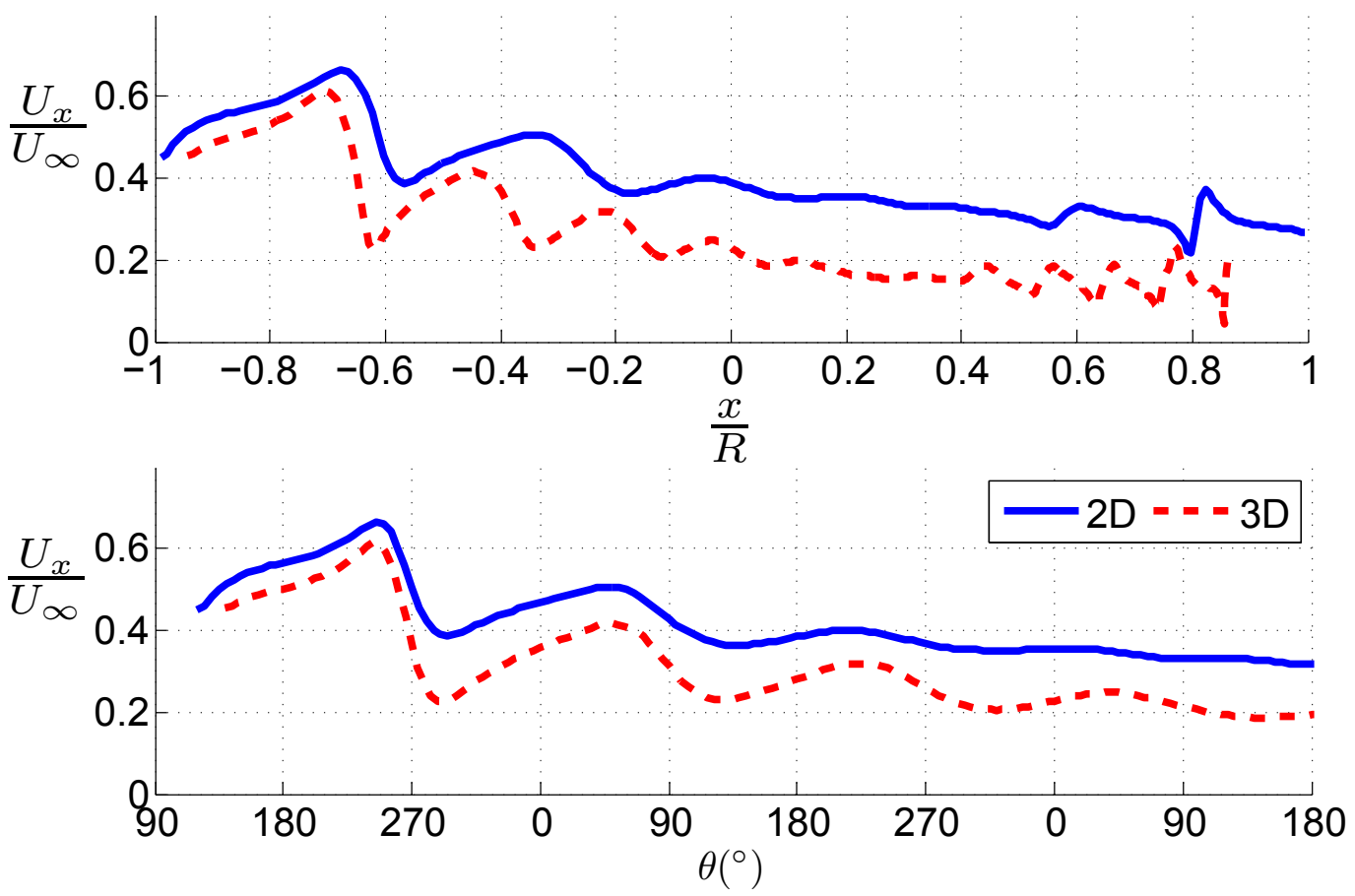

Figure 26: Evolution of the velocity along the $x$-axis $(y / R=0, z / H=0)$, comparison of $2 D$ and $3 D$ simulations. 


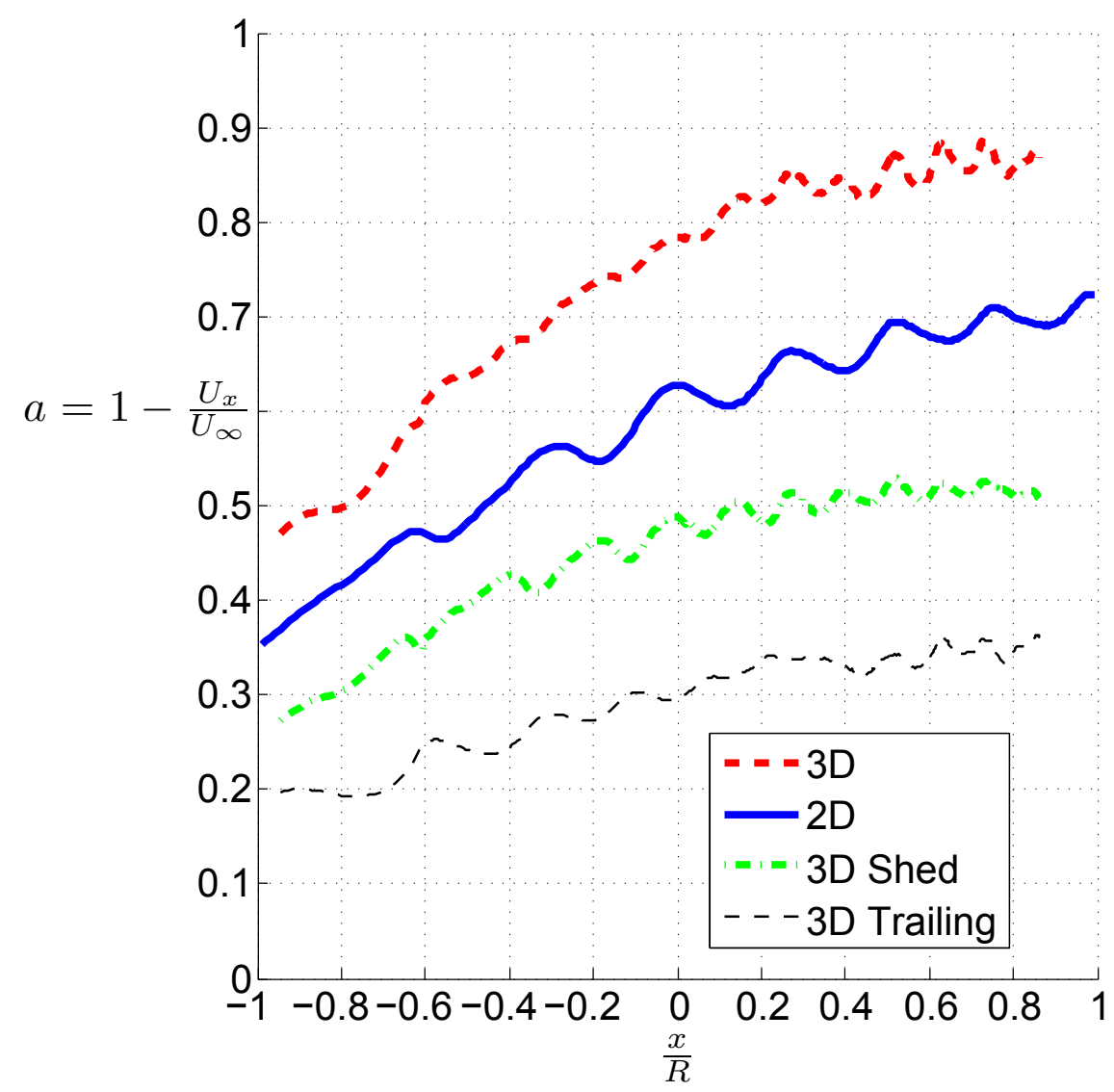

Figure 27: Evolution of the induction (wake induction only) at $x$-axis $(y / R=0, z / H=0)$, comparison of $2 D$ and $3 D$ simulations. 
$3 D$ models, together with the composition of the $3 D$ induction in the induction due to shed and trailing vorticity (the effect of the bound circulation of the blades is neglected).

The results of Figure 27 show clearly that the induction from shed vorticity in the $3 D$ flow is lower than the induction in the $2 D$ flow, which comprises only shed vorticity.

The results show that the higher induction in the $3 D$ flow is a result of the additional effect of the trailing vorticity. Thus, the spanwise distribution of circulation on the blade has an impact not only in the induced drag of the blade and loads, but also on the convection of the wake.

This lower convection speed of the wake in the $3 D$ flow creates a wake equivalent to that of an higher tip-speed ratio $2 D$ flow, resulting in a larger induction on the blades and a consequent loss of efficiency.

Despite the more closely packed set of wake sheets, the $3 D$ induction due to shed vorticity is slightly lower than the $2 D$ case. This is expected, since the $2 D$ case corresponds to an infinite span case, and also to a higher range of bound vorticity over the rotation.

Another interesting effect, albeit second order, is the previously-mentioned impact of the release of new wake on the oscillation of the velocity of the wake. The results in Figure 27 show the oscillations also present in the $2 D$ data, and more interestingly, the show opposition of phase of the oscillation between the induction due to shed vorticity and trailing vorticity in the $3 D$ flow. This opposition of phase is a consequence of the opposition of phase during the rotation of the generation of shed and trailing vorticity.

During the convection inside the rotor $(-1<x / R<1)$, the $3 D$ flow case experiences a larger number of oscillations in acceleration than the $2 D$ flow; this is due to the increased effective local tip-speed ratio experienced by the $3 D$ wake.

\section{B. $2 D$ vs $3 D$ bound circulation and normal force}

Figure 28 shows the blade's bound vorticity calculated with the $2 D$ and the $3 D$ unsteady free-wake panel models; the $3 D$ results correspond to the midsection of the blade.

The results of Figures 26 and 27 showed the larger induction in the $3 D$ case in comparison with the $2 D$. This larger induction comes not only from the presence of the induction due to trailing vorticity, but also from a more closely packed set of wakes. This larger induction results in a slightly lower value of bound vorticity for the $3 D$ flow case during the upwind half of the rotation $\left(0^{\circ}<\theta<180^{\circ}\right)$.

However, during the downwind blade passage $\left(180^{\circ}<\theta<360^{\circ}\right)$, the $3 D$ effect of induction is even more relevant and the difference between $2 D$ and $3 D$ bound vorticity is larger. As discussed in Section $\mathrm{X}$, due to the large induction during the downwind blade passage, the magnitude of the circulation due to the pitching motion is larger than the circulation due to the local wind (which has an opposite sign).

The $3 D$ effect of induction has an even more significant impact on the load distribution. Figure 29 shows the normal force on the blade section calculated with the $2 D$ and the $3 D$ unsteady free-wake panel models; the $3 D$ results correspond to the midsection of the blade.

Thus, the $3 D$ induction affects loads twice: by varying the value of bound vorticity and also by varying the local velocity field.

\section{The 3D effect of the pitching motion of the VAWT blade.}

As mentioned earlier, the pitching motion of the blade during rotation results in an added bound circulation. In a non-deformable blade, this added circulation is constant and thus does not, in $2 D$ potential flow, affect the shed vorticity (this is not true in viscous flow); it does, however, affect the wake and has an effect on the exchange of energy.

In $3 D$ flow, the bound circulation due to pitching affects the trailing vorticity. The spanwise variation of circulation due to the pitching motion is a result of a spanwise variation of the chord and/or pitching axis location.

A spanwise variation of bound circulation generates the release of trailing vorticity. For the VAWT, this extra trailing vorticity has three main effects:

- Varying the strength of the tip vortex, resulting in a change of the tip vortex roll-up process, and thus its convection in/outboard.

- Changing the induction on the blade, thus changing the energy conversion and induced drag. 


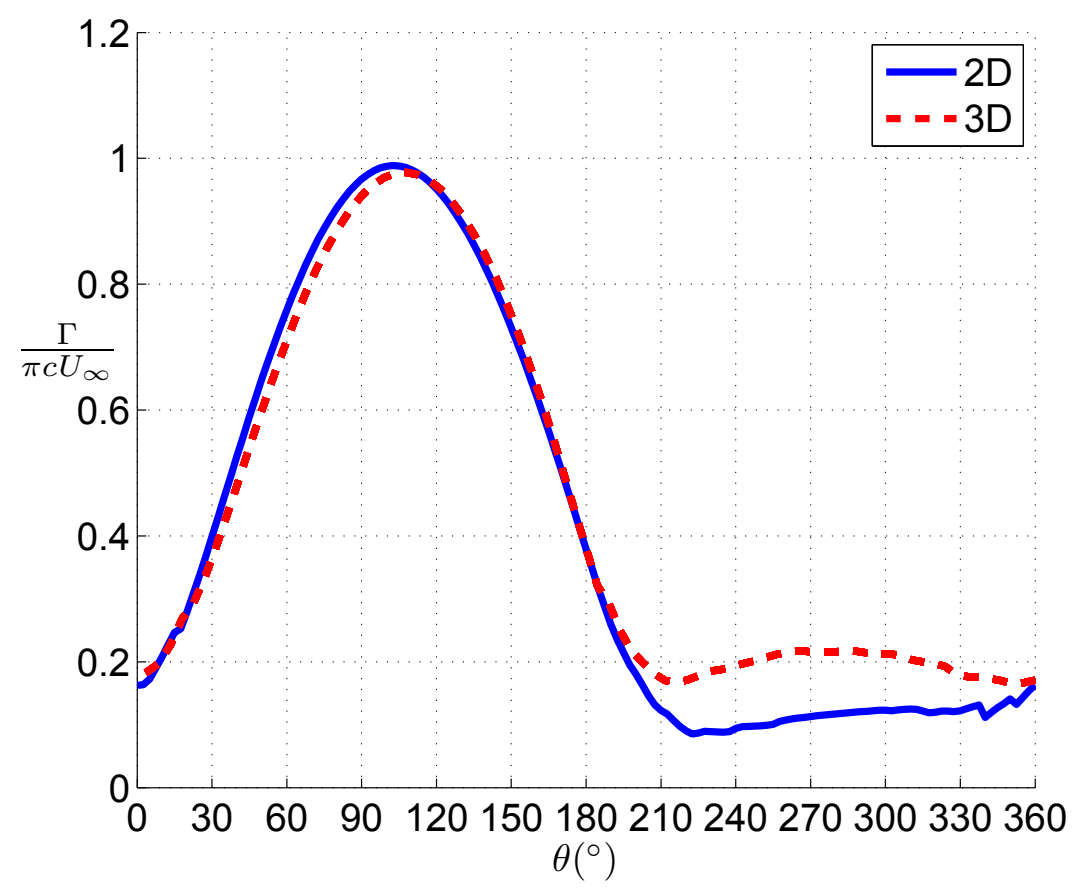

Figure 28: $2 D$ and $3 D$ bound vorticity during the rotation.

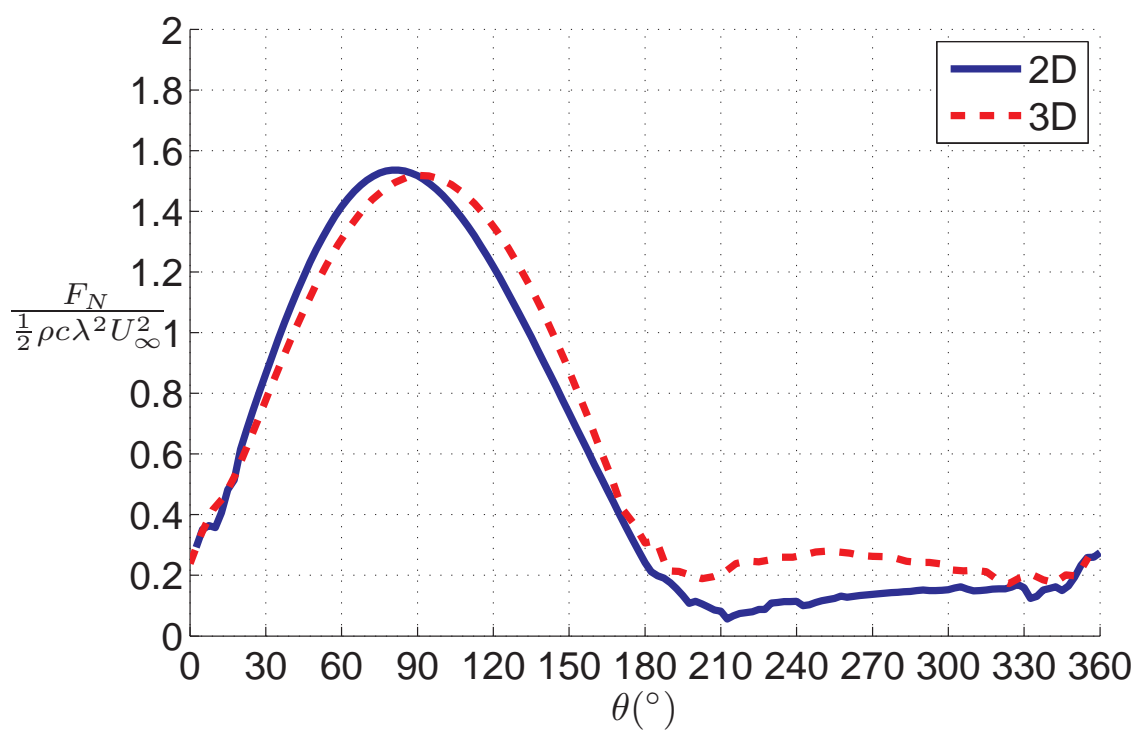

Figure 29: $2 D$ and $3 D$ normal force over the blade during the rotation.

30 of 33 
- Varying the strength of the trailing vorticity and the self-induction of the wake, leading to a lower/larger effective tip-speed ratio, as discussed in Section XI.

In order to illustrate the need to account for the distribution of circulation due to the pitching motion of the blade, Figure 30 plots the near wake of a two-bladed, straight, constant-chord rotor, in a $U_{\infty}=0, \lambda=\infty$ condition (no wind). The wake strength is driven by the trailing vorticity due to the spanwise distribution of the bound circulation. This bound circulation consists of the circulation due to the pitching motion of the blade and the circulation due to the inflow of the wake induction. The tip vortex moves inboard, due to its roll-up evolution. The VAWT experiences an induced drag due just to its rotation, even in $U_{\infty}=0$ conditions.

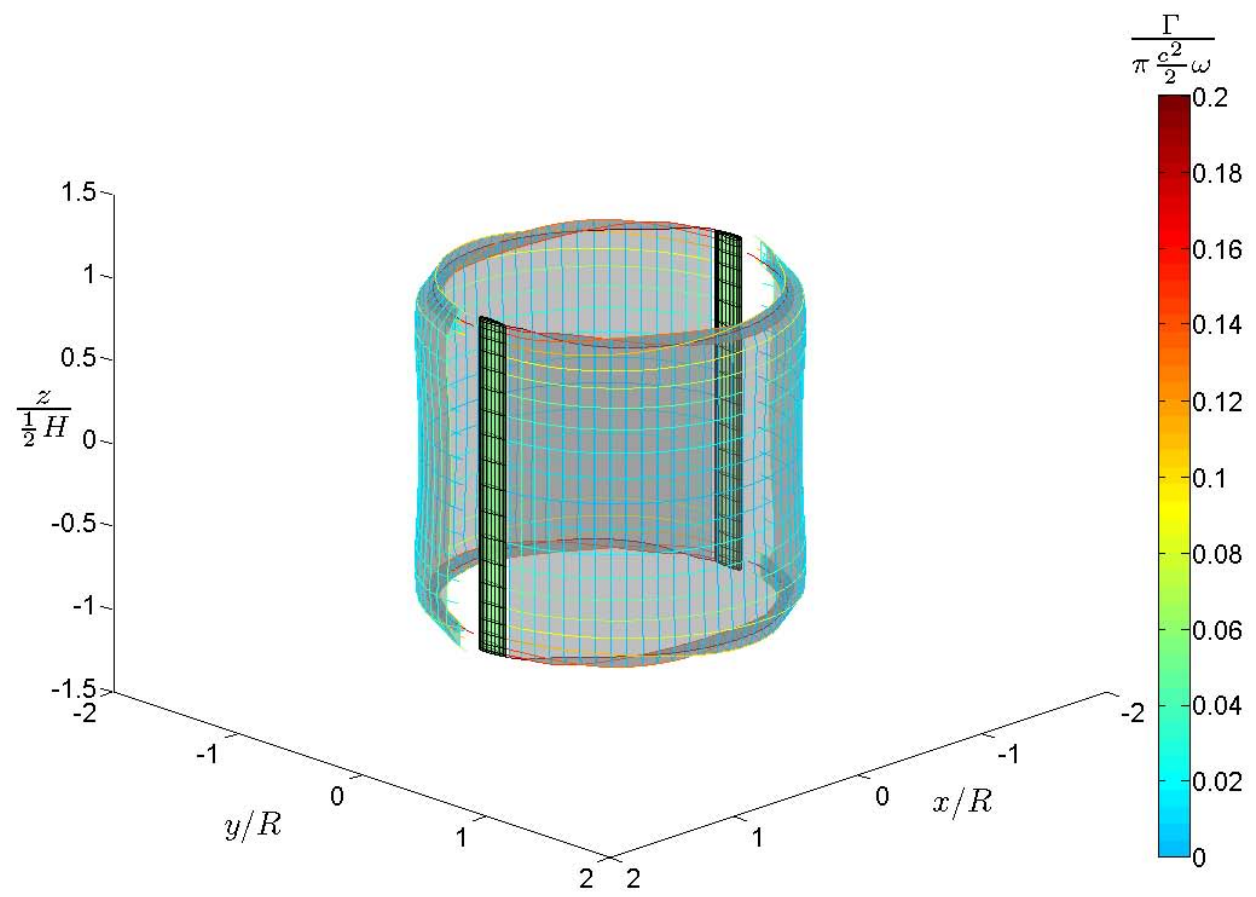

Figure 30: Wake evolution and roll-up in a $U_{\infty}=0$ condition for a two-bladed VAWT.

Thus, when designing the $3 D$ blade for reducing the induced drag and the effect of the tip vortex, one must account for the distribution of circulation due to the steady pitching motion of the blade.

\section{Conclusions}

The spanwise distribution of circulation on the H-VAWT blade leads to the release of trailing vorticity, of which the tip vortex is the most prominent element. The observation of the tip-vortex convection and the wake expansion of Horizontal Axis Wind Turbines has led researchers to assume that the expansion of the H-VAWT also follows the radial expansion observed in the HAWT. However, since the expansion of the wake of the VAWT occurs in the $2 D$ plane, there is no requirement for a spanwise expansion.

The use of (Stereo) Particle Image Velocimetry allows a more detailed visualization and quantification of the tip-vortex convection and evolution (expansion/dissipation). The measurements are performed in two directions of field of view (measurement plane): normal to the spanwise direction of the blade (xy-planes), at several positions of the blade tip; and parallel to the spanwise direction and $U_{\infty}$ ( $x z$-planes), at several $y$ positions.

The data from the first orientation of the planes are used for the reconstruction of the tip-vortex tube core; this enables the visualization of the curvature of the tube, due to its convection in the $x$-direction and 
the $z$-direction. The curvature of the tip-vortex tube confirms the tip-vortex dynamics observed with the hot-wire measurements.

The $3 D$ reconstruction of the vorticity field in the tip-vortex region enables the identification of not only the tip vortex, but also of the adjacent wake sheet, as it is rolled up by the tip vortex.

The second direction of the measurement planes, parallel to the spanwise direction and $U_{\infty}(x z$-planes), presents slices of the tip-vortex tube. These slices allow a clearer visualization of the tip-vortex core and the wake sheet rolling around it (Figures 7 to 10 ).

The convection of the tip vortex at the different $x z$-planes shows once again the inboard/outboard motion of the tip vortex as a function of $y / R$. The data also enables the quantification of circulation in the tip vortex and its convection and quantification of the resultant induction field, all of which will be used for model validation.

The measurements of the different strength of tip vortices generated by the different blade tip shapes confirms the impact of chord distribution and pitching axis location on the local distribution of the bound circulation and thus, the distribution of the trailing vorticity.

The $3 D$ near wake of the H-VAWT can be divided in four regions where different dominant effects are playing:

- The upwind blade passage, where the flow is determined by an almost planar approach of the induction field, similar to an actuator disk approach

- The leeward and windward blade passage regions, where the flow is dominated by the induction of the wake segments of different revolution, that due to their proximity generate strong roll-up of the wake at the tip region. The "new wake inside old wake" phenomenon defines the difference between the leeward and windward regions in terms of expansion.

- The evolution inside the rotor volume of the wake generated during the upwind blade passage, with the inboard motion of the tip vortex.

- The downwind blade passage, and the interaction with the upwind generated wake trailing vorticity/tip vortex.

The finite blade and the induction by the trailing vorticity clearly make the rotor less efficient then the $2 D$ rotor. This inefficiency can be expressed by the spanwise gradient of the shed vorticity, which mirrors the power conversion between rotor and flow.

The convection of the wake inside the rotor volume is no longer a $2 D$, but clearly determined by the trailing vorticity of the $3 D$ flow. Although the wake convection in the $3 D$ simulation is similar to what is seen in the $2 D$ flow case, this is due to the fact that, in the $3 D$ flow case, the lower induction due to the weaker shed vorticity is compensated (and exceeded) by the induction due to the trailing vorticity. The $3 D$ rotor, at the midspan region, has a higher induction in $x$-direction than the $2 D$ case. This was confirmed with the experimental data of the PIV flow field measurement in the mid span region, where the $3 D$ wake model better predicts the location of the wake, once again confirm the $3 D$ behavior of the wake convection, even at the midspan.

This higher induction will result in a more packed set of wakes, meaning that the spacing between wakes is similar to what is found at higher tip speed ratios in the $2 D$ case. This implies a higher induction on the blade, both during the upwind and and specially during the downwind blade passage, once again decreasing the efficiency of the rotor.

Further results and a more complete discussion of some of these points can be found in the works of, ${ }^{1210}$ and. ${ }^{9}$

\section{Bibliography}

\section{References}

${ }^{1}$ I. Paraschivoiu. Wind turbine design with emphasis on Darrieus Concept. Polythecnic International Press, 2002.

${ }^{2}$ T.D. Ashwill and T.H. Leonard. Developments in the blade shape design for a Darrieus vertical axis wind turbine. Technical Report SAND86-1085, Sandia National Laboratories, 1986.

${ }^{3}$ B.F. Blackwell and G.E. Reis. Blade shape for troposkien type of vertical-axis wind turbine. Technical Report SAND740154, Sandia National Laboratories, 1974. 
${ }^{4}$ D.E. Berg. Structural design of the Sandia 34-meter vertical axis wind turbine. Technical Report SAND84-1287, Sandia National Laboratories, 1984.

${ }^{5}$ T.G. Carne and A.R. Nord. Modal testing of a rotating wind turbine. Technical Report SAND82-0631, Sandia National Laboratories, 1982.

${ }^{6}$ T.G. Carne, D.W. Lobitz, A.R. Nord, and R.A. Watson. Finite element analysis and modal testing of a rotating wind turbine. Technical Report SAND82-0345, Sandia National Laboratories, 1982.

${ }^{7}$ Brian Kinloch Kirke. Evaluation of Self-Starting Vertical Axis Wind Turbines for Stand-Alone Applications. PhD dissertation, Griffith University, Faculty of Engineering and Information Technology, School of Engineering, 1998.

${ }^{8}$ S. Mertens. Wind energy in the built environment: concentrator effects of buildings. PhD thesis, Delft University of Technology, 2006.

${ }^{9}$ C. Hofemann. Investigation on tip vortex evolution of a vertical axis wind turbine with Stereo PIV. Master's thesis, Faculty of Aerospace Engineering, Delft University of Technology, May 2008.

${ }^{10} \mathrm{~K}$. Dixon. The near wake structure of a vertical axis wind turbine -including the development of a 3D unsteady free-wake panel method for VAWTs. Master's thesis, Faculty of Aerospace Engineering, Delft University of Technology, April 2008.

${ }^{11}$ K. Dixon, C.J. Simão Ferreira, C. Hofemann, G.J.W. van Bussel, and G.A.M. van Kuik. A 3D unsteady panel method for vertical axis wind turbines. In T Chaviaropoulos, editor, The proceedings of the European Wind Energy Conference and Exhibition EWEC 2008 Brussels, 2008.

${ }^{12}$ C.J. Simão Ferreira. The near wake of the VAWT: $2 D$ and $3 D$ views of the vawt aerodynamics. PhD thesis, Delft University of Technology, October 2009. 